\title{
A Homogenized Energy Framework for Ferromagnetic Hysteresis
}

\author{
Ralph C. Smith \\ Center for Research in Scientific Computation \\ Department of Mathematics \\ North Carolina State University \\ Raleigh, NC 27695-8205 \\ rsmith@eos.ncsu.edu \\ Thomas R. Braun \\ Center for Research in Scientific Computation \\ Department of Mathematics \\ North Carolina State University \\ Raleigh, NC 27695-8205 \\ trbraun@unity.ncsu.edu
}

\author{
Marcelo J. Dapino \\ Department of Mechanical Engineering \\ The Ohio State University \\ Columbus, OH 43210 \\ dapino.1@osu.edu
}

\author{
Anthony P. Mortensen \\ Department of Mechanical Engineering \\ The Ohio State University \\ Columbus, OH 43210 \\ mortensen.19@osu.edu
}

\begin{abstract}
In this paper we develop a macroscopic framework quantifying the hysteresis and constitutive nonlinearities inherent to ferromagnetic materials. In the first step of the development, we construct Helmholtz and Gibbs energy relations at the mesoscopic or lattice level based on the assumption that magnetic moments or spins are restricted to two orientations. Direct minimization of the Gibbs energy yields local average magnetization relations appropriate for operating regimes in which relaxation mechanisms are negligible whereas the balance of the Gibbs and relative thermal energies through Boltzmann principles provides local models which incorporate mechanisms such as thermal after-effects. To construct macroscopic relations that incorporate material nonhomogeneities, polycrystallinity, and variable effective fields, we employ stochastic homogenization techniques based on the assumption that parameters such as local coercive and interaction fields are manifestations of underlying distributions. The resulting framework quantifies in a natural manner the anhysteretic magnetization provided by decaying $\mathrm{AC}$ fields and guarantees the closure of biased minor loops once transient accommodation and after-effects are complete. Furthermore, noncongruency is achieved with certain choices for the energy functionals. Hence the framework provides an energy basis for certain extended Preisach models and the relation of the framework to several macroscopic hysteresis models is detailed. The behavior of both the nonlinear anhysteretic relations and full hysteresis model are validated through comparison with experimental steel and nickel data.
\end{abstract}




\section{Introduction}

The modeling of hysteresis in ferromagnetic materials is a classical problem, dating back to at least Maxwell [1], which has profound implications for present and projected applications utilizing magnetic materials. As the range of magnetic applications grows, so too does the list of requirements necessary for the models used to quantify hysteresis and constitutive nonlinearities inherent to the compounds. For example, transducer design, material characterization, optimization of magnetic recording media, and development of magnetic computing paradigms require high fidelity models that are feasible to implement in optimization algorithms. Real-time implementation of model-based control algorithms for such systems necessitates the development of low-order macroscopic models which are easily constructed and updated to accommodate changing environmental conditions. To optimize material design, however, it is necessary to construct models at the microscopic and mesoscopic levels to quantify and predict the effects of changing material composition. Moreover, these microscopic models must be commensurate with macroscopic measurements to permit evaluation and updating of the material designs. This necessitates the development of multiscale modeling hierarchies in which energy-based fine-scale models are used to predict effective parameters in higher level models.

As a prelude to constructing any energy-based models, it is necessary to at least qualitatively understand the physical mechanisms which produce hysteresis and constitutive nonlinearities. In magnetic materials, these properties are due to a number of mechanisms but the primary sources of hysteresis are moment or domain interactions, domain wall losses caused by material inclusions, and material anisotropies [2-5]. Micromagnetic models can accommodate a number of these mechanisms but do so at high computational cost. There are presently no macroscopic models which quantify all of these effects for general, broadband, variable temperature, variable stress operating conditions, and present macroscopic theories address one or two of these mechanisms as dictated by the regimes under consideration.

In this paper, we develop a macroscopic framework which quantifies hysteresis and constitutive nonlinearities in the $H-M$ and $H-B$ relation for a variety of ferromagnetic materials and yields anhysteretic magnetization relations in a natural manner. It guarantees the closure of biased minor loops once after-effect and accommodation processes are complete but does not enforce congruency in accordance with noncongruencies measured in certain operating regimes. The model incorporates relaxation mechanisms but neglects eddy current losses so it should be employed for low frequency material characterization or architectures for which eddy current losses are minimal (e.g., laminated Terfenol-D rods). It is constructed in the context of uniaxial moment configurations but does not incorporate additional anisotropy energy mechanisms; hence it applies to isotropic polycrystalline materials, a variety of uniaxial regimes, and weakly anisotropic materials. Finally, the characterization framework is sufficiently efficient to permit model-based system and control design.

In the first step of the model development, a mean field approximation for the exchange energy of moments on a uniform lattice is balanced with entropy effects to construct a Helmholtz free energy relation. This in turn is used to construct a piecewise quadratic Helmholtz energy which retains salient features of the statistical mechanics model at fixed temperatures but is more efficient to implement. The incorporation of the magnetoelastic energy subsequently yields a Gibbs energy relation which quantifies changes in the energy due to an applied field. For general operating regimes, the Gibbs and relative thermal energies are balanced through Boltzmann probability relations to yield a local average magnetization model which incorporates the thermal activation mechanisms which produce thermal after-effects [2] and certain manifestations of accommodation or reptation [6]. In the limit of negligible thermal activation, it is proven that these relations reduce to the local magnetization kernels, or hysterons, obtained by minimizing the Gibbs energy. For certain regimes, 
it is illustrated that the kernels are given by Ising relations. In the second step of the development, we incorporate lattice nonhomogeneities, inclusions, polycrystallinity, texture and certain mean field effects by assuming that quantities such as the local coercive and effective fields are manifestations of underlying distributions rather than fixed parameters. Stochastic homogenization in this manner yields macroscopic models suitable for material characterization.

To place the modeling framework in perspective, we compare it with four presently employed macroscopic models: Jiles-Atherton, Stoner-Wohlfarth, Globus and Preisach - detailed comparison of these models can be found in [7]. These four approaches were chosen to illustrate similarities and differences with the proposed framework, and they represent what can now be considered as established macroscopic hysteresis models. However, they are by no means exhaustive and additional hysteresis models can be found in $[2-4,8,9]$. Additionally, details about micromagnetic theory and its relation to the proposed model is included in Section 2 where it is more in context.

\section{Jiles-Atherton Model}

The Jiles-Atherton model characterizes hysteresis in isotropic materials through the quantification of domain wall losses [4,10-12]. In its original formulation, the model was constructed in two steps: (i) quantification of the anhysteretic magnetization, and (ii) incorporation of irreversible and reversible domain wall effects. The anhysteretic magnetization is modeled through the Langevin relation

$$
\begin{aligned}
& M_{a n}=M_{s}\left[\operatorname{coth}\left(H_{e} / a\right)-\left(a / H_{e}\right)\right] \\
& H_{e}=H+\alpha M
\end{aligned}
$$

where $M_{s}$ is the saturation magnetization, $\alpha$ is a mean field parameter, and $a$ is a parameter having dimensions of field. The irreversible and reversible domain wall losses are incorporated by determining the magnetostatic energy required to reorient moments in the presence of the effective field $H_{e}$. In this manner, the model also incorporates certain rotational effects.

The original model has subsequently been extended to include eddy current losses [13], certain anisotropies [14] and to enforce closure of biased minor loops. The minor loop extension provided by Carpenter [15] is phenomenological in the sense that it is based on translates and scaling of the major loop curves whereas the extension provided by Jiles [16] relies on a priori knowledge of future turning points. This reduces the utility of the model for dynamic control applications where turning points are determined by a feedback law responding to state measurements and hence are unknown before the control commences.

It will be observed that the proposed model yields a hysteresis kernel at the mesoscopic level which is formulated as the Ising relation $\bar{M}=M_{s} \tanh \left(H_{e} / a\right)$ and hence agrees through first-order terms with (1). The primary difference between the theories lies in underlying energy formulation and manner through which losses are incorporated.

\section{Stoner-Wohlfarth Model}

The original Stoner-Wohlfarth model quantified the rotation of noninteracting, single-domain particles having uniaxial anisotropy [17]. While the model has received widespread use in the magnetic recording industry, its use for general material characterization was originally limited by the fact that it did not incorporate moment interactions. A number of recent extensions to both the model and underlying philosophy have substantially improved its utility. The model was extended to include cubic anisotropies by Lee and Bishop [18] whereas Armstrong [19], Clark et al. [20], and Jiles and Thoelke [21] extended the model to quantify magnetoelastic effects in Terfenol-D. Certain mean field effects are incorporated in [22,23] while pinning losses are incorporated in [7] where it is 
illustrated that this latter mechanism is necessary to achieve physical minor loop behavior. Finally, relations between the Stoner-Wohlfarth model and micromagnetic models are detailed in [8].

The hysteresis kernels, or hysterons, provided by the proposed model are analogous to those predicted by the Stoner-Wohlfarth model when the applied magnetic field is aligned with the easy axis of particles. This is consistent with the assumption in the proposed framework that moment alignment occurs in two diametrically opposite directions. The assumptions differ from the original Stoner-Wohlfarth model in that moment interactions are incorporated whereas anisotropy energies are neglected.

\section{Globus Model}

The Globus model preceded that of Jiles of Atherton and bears certain similarities in that it quantifies irreversible and reversible hysteresis effects through domain wall mechanisms [24]. A primary difference between the theories lies in the Globus assumption that domain walls are pinned on grain boundaries which results in the simplifying assumption that reversible domain wall bending and irreversible domain wall displacement can be considered on a single, representative spherical grain. While efficient to implement, this model lacks a number of mechanisms required for high performance applications.

The anhysteretic curves provided by the Globus model are analogous to the mesoscopic hysteresis kernel provided by the new theory. However, the latter includes moment interactions and physical minor loop behavior which makes it advantageous for general applications.

\section{Preisach Framework}

Preisach's original model quantified the hysteretic relation between input fields $H(t)$ and the magnetization $M(t)$ through a superposition of rectangular hysteresis relays or kernels $\left[k_{s_{1}, s_{2}}(H)\right](t)$ [25]. Here $s_{1}$ and $s_{2}$, with $s_{1}<s_{2}$, provide thresholds at which the kernel switches between +1 and -1 . The magnetization is modeled as

$$
[M(H)](t)=\int_{0}^{\infty} \int_{-\infty}^{\infty} \nu(r, s)\left[k_{s-r, s+r}(H)\right](t) d s d r
$$

where $\nu$ is a density, or weighting function which depends on properties of the material under consideration [26]. It is illustrated in $[7,9]$ that one choice of $\nu$ is the normal density function

$$
\nu\left(h_{I}, h_{c}\right)=\frac{M_{s}}{2 \pi \sigma_{c} \sigma_{I}} e^{-\left(h_{c}-\bar{h}_{c}\right)^{2} / 2 \sigma_{c}^{2}} e^{-h_{I}^{2} / 2 \sigma_{I}^{2}}
$$

where $h_{I}$ and $h_{c}$ denote interaction and coercive fields, $\bar{h}_{c}$ is an average coercive field, and $\sigma_{I}, \sigma_{c}$ are respective variances. For general characterization, the weight or measure $\nu$ can be estimated from measured data through techniques analogous to those described in [27,28].

The advantage of the Preisach methodology lies in its generality. From a solely mathematical perspective, it can be used to characterize material behavior in regimes where the underlying physics is poorly understood or unknown. It also yields models which can, at least approximately, be inverted for linear control design [28]. However, these advantages are complemented by a number of disadvantages. First, the nonphysical nature of the measures or parameters makes it difficult to construct models using known physical behavior or to employ attributes of the data for updating models to accommodate changing operating conditions. While extensions to the Preisach model have recently been proposed to facilitate parameter identification through correlation with physical mechanisms $[9,29]$, the accurate characterization of biased minor loops typically requires general weights comprised of a large number of nonphysical parameters. A more fundamental difficulty 
arises in operating regimes involving broadband input signals and temperature or load dependence since the classical Preisach model is based on the assumptions of frequency, load and temperature invariance. Finally, classical Preisach models erroneously enforce congruency in certain regimes and do not accommodate reversible magnetization mechanisms. As detailed in $[6,7,9,30]$, extensions to the classical theory have been developed to address a number of these issues. However, these extensions add significant complexity to the theory and reduce its efficiency for real-time implementation. For example, one technique for incorporating temperature-dependence is to employ vector-valued measures or weights $[\nu(r, s)](T)$ (e.g., see [31]). For control design, however, this necessitates the use of lookup tables which reduces significantly the efficiency of associated control algorithms.

As illustrated in $[32,33]$, the proposed model can be interpreted as providing an energy basis for certain extended Preisach models. In summary, the kernels derived through energy considerations at the mesoscopic level provide the Preisach hysterons whereas the stochastic densities, used to obtain macroscopic models, provide the Preisach weights. However, the proposed framework differs from the classical Preisach model in five crucial aspects. (i) The first is the energy basis of the model - formulation through energy analysis provides a low-order model which, for certain density choices, has parameters that can be physically correlated with properties of the data. (ii) Due to the energy basis of the framework, stress and temperature-dependencies are incorporated in the basis or kernel in the new model (e.g., see $[34,35]$ ) whereas they enter the weights in the Preisach formulation. Because they are incorporated in the kernel, the model automatically incorporates these effects which eliminates the necessity of vector-valued weights or lookup tables. From the perspective of implementation, this indicates that only one set of parameters must be identified for the proposed model and no switching between parameter sets is required during operation. This significantly augments the efficiency of characterization and control algorithms employing the model. (iii) The incorporation of relaxation mechanisms through the energy basis provides the framework with the property that it accommodates nonclosure or nondeletion properties in accordance with measured material properties. In this case, the framework provides an energy basis for certain extended Preisach formulations based on Arrhenius relations [9]. (iv) Derivation of the theory from Boltzmann principles yields kernels or hysterons which accommodate the noncongruency observed for certain materials and operating regimes. This is in contrast to input and output-dependent (moving) Preisach formulations which incorporate noncongruency through input or output-dependent densities. (v) The model automatically incorporates reversible magnetization mechanisms for small AC field excursions about a fixed DC value - hence it accurately characterizes reversible material behavior following field reversal without the extensions required for Preisach formulations.

The proposed framework builds upon and significantly extends ferromagnetic theory originally developed in [36]. The extensions include the derivation of energy relations based on statistical mechanics tenets, formulation of the model in terms of general densities, and rigorous analysis establishing the convergence of models incorporating thermal activation to those derived through minimization of the Gibbs energy as relative thermal energies become negligible. In the present work, we also establish the manner through which the anhysteretic magnetization is characterized and summarize highly efficient implementation algorithms.

Appropriate Helmholtz and Gibbs energy relations are constructed at the lattice level in Section 2 and used in Section 3 to quantify the local average magnetization $\bar{M}$ and local anhysteretic magnetization $\bar{M}_{a n}$ in the presence and absence of thermal activation. These local relations are combined with stochastic homogenization techniques in Section 4 to construct macroscopic models which quantify the hysteresis and constitutive nonlinearities inherent to a variety of ferromagnetic compounds. The properties of both the anhysteretic and full hysteresis models are illustrated in Section 5 through numerical simulations and comparison with experimental steel data. 


\section{Energy Relations for Homogeneous Materials}

The microscopic and macroscopic behavior of ferromagnetic materials is defined by the exchange, anisotropy, magnetostatic and magnetoelastic energies, and hence a complete energy-based theory quantifying hysteresis and magnetic properties of these materials must include, or at least, accommodate these contributions. The magnetostatic energy quantifies long range interactions between magnetic moments and applied fields and is significant in both ferromagnetic and paramagnetic materials. Additionally, ferromagnetic materials exhibit exchange interactions between neighboring atomic spins which serves to align moments. This effect is short range and typically influences nearest, or next-nearest, neighbors. The exchange interactions and associated exchange energy also differentiate ferromagnetic and paramagnetic compounds. The anisotropy energy quantifies changes in the internal energy of materials due to changes in the direction of the magnetization. Whereas magnetic anisotropy can be due to a number of factors, we will focus primarily on crystalline and stress anisotropies. Finally, the magnetoelastic energy quantifies magnetomechanical coupling inherent to the materials.

The first statistical mechanics model for the exchange interactions was posed by Ising in 1925 and was based on the assumption of a linear lattice of magnetic moments in which only neighbors interact [37]. This was later extended by Heisenberg in 1928 to include quantum effects and complete correlation between neighboring electrons having overlapping wave functions [38]. In the Heisenberg model, the interaction energy between spins $\mathbf{S}_{i}$ and $\mathbf{S}_{j}$ is

$$
U=-2 \mathcal{J} \mathbf{S}_{i} \cdot \mathbf{S}_{j}
$$

where $\mathcal{J}$ is an exchange integral with $\mathcal{J}>0$ for ferromagnetic materials and $\mathcal{J}<0$ for antiferromagnetic compounds. The exchange energy for the system is then obtained by summing over all magnetic moments which yields

$$
U=-2 \sum_{i j} \mathcal{J}_{i j} \mathbf{S}_{i} \cdot \mathbf{S}_{j}
$$

The Ising model can be obtained from that of Heisenberg by truncating the interaction energy for the lattice. For example, if quantization is assumed to take place only in the $z$-direction (e.g., the direction of an applied field $\mathbf{H}$ ), the full inner product $\mathbf{S}_{i} \cdot \mathbf{S}_{j}=S_{i x} S_{j x}+S_{i y} S_{j y}+S_{i z} S_{j z}$ is replaced in the Ising model by the $z$-component $S_{i z} S_{j z}$. If the restricted spins are denoted by $\sigma_{i}= \pm 1$, the Ising relation for the exchange energy can be formulated as

$$
U=-2 \sum_{\langle i j\rangle} \mathcal{J}_{i j} \sigma_{i} \sigma_{j}
$$

where the notation $\langle i j\rangle$ indicates summation over nearest neighbors. In addition to the simplification provided by reduced dimensionality, the Ising model admits a classical treatment of the spins, due in part to the fact that spins commute in the truncated expansion and hence can be treated as variables, whereas spins must be treated as quantum mechanical operators in the Heisenberg model. While the Ising model proves sufficiently accurate when characterizing the exchange energy in a number of applications, it is necessary to include the quantum effects incorporated in the Heisenberg model to quantify mean field properties from first principles.

As detailed in $[8,39]$, the Heisenberg energy relation (5) is exactly solvable for only a few cases, one of which is the Ising model, and is completely isotropic. The inclusion of the anisotropy and magnetostatic energy in the Heisenberg Hamiltonian or energy formulation yields a model that is prohibitively expensive to approximate due to its generality. This necessitates the consideration of simplified theories motivated by the quantum energy relations but tractable for implementation. 
One technique in this vein is the theory of micromagnetics which originated with the work by Landau and Lifshitz in 1935 [40] on the analysis of domain walls. Significant contributions to the theory were provided by Brown [41-43] and subsequent researchers; e.g. see [8]. The basic tenet in this approach is to employ classical theory in combination with quantum principles to predict the distribution of spins through the minimization of general energy relations

$$
U=U_{E}+U_{A}+U_{\lambda}+U_{M}
$$

where $U_{E}, U_{A}, U_{\lambda}$ and $U_{M}$ respectively denote the exchange, anisotropy, magnetoelastic and magnetostatic energies. The capability this theory provides for predicting domain structures in ferromagnetic materials is illustrated in [44] where a micromagnetic energy relation of the form (7), with the specific energy components defined by

$$
\begin{aligned}
U_{E} & =-2 \sum_{x} \sum_{y} \sum_{z} \mathcal{J} \mathbf{S}_{i} \cdot \mathbf{S}_{j} \\
U_{A} & =-2 \sum_{x} \sum_{y} \sum_{z}\left[K_{1}\left(\alpha_{1}^{2} \alpha_{2}^{2}+\alpha_{2}^{2} \alpha_{3}^{2}+\alpha_{3}^{2} \alpha_{1}^{2}\right)+K_{2} \alpha_{1}^{2} \alpha_{2}^{2} \alpha_{3}^{2}\right] \\
U_{\lambda} & =-\frac{3}{2} \sum_{x} \sum_{y} \sum_{z} \lambda \sigma \cos ^{2} \phi \\
U_{M} & =-\frac{1}{2} \sum_{x} \sum_{y} \sum_{z} \mathbf{m}_{i} \cdot \mathbf{H}_{I}
\end{aligned}
$$

was minimized on a Cray X-MP/22 for a cubic grid consisting of $22 \times 22 \times 22$ exchange coupled spins. Here $K_{1}$ and $K_{2}$ are anisotropy constants and $\alpha_{1}, \alpha_{2}$ and $\alpha_{3}$ are direction cosines of a moment $\mathbf{m}_{i}$ at the center of the cube. Furthermore, $\lambda, \sigma$ and $\phi$ respectively denote the magnetostriction constant, magnetoelastic stress and angle between the magnetization and stress. Finally, $\mathbf{H}_{I}$ denotes the interaction field at $\mathbf{m}_{i}$ due to all moment.

In addition to the capabilities that this theory provides for predicting the domain structure and dynamics of ferromagnetic materials, it is established in [8] that this approach provides a set of differential equations for which the Stoner-Wohlfarth model is an eigenfunction or mode.

However, due to their complexity, models derived through micromagnetic principles presently preclude real-time implementation. The predictive capabilities provided by the models include more detail than is necessary for modeling hysteresis in a manner that facilitates system and control design. To provide such quasi-macroscopic models, we construct an energy formulation which incorporates certain aspects of the Ising and micromagnetics models at the microscopic scale but employs statistical techniques rather than physical principles to obtain a commensurate macroscopic magnetization model.

\subsection{Helmholtz Energy}

We consider two techniques for specifying the Helmholtz energy at the lattice level. The first is based on statistical mechanics principles and hence includes certain temperature dependencies; the second is obtained through an approximation of the first for fixed temperature regimes.

The statistical mechanics model is based on an approximation to the Ising model which has the requisite assumption that spins or magnetic moments are restricted to two possible orientations, $\sigma_{i}= \pm 1$.

The assumption that spins have two preferred orientations appears at first to be highly restrictive but can in fact be physically motivated for a number of regimes. From a classical perspective, 

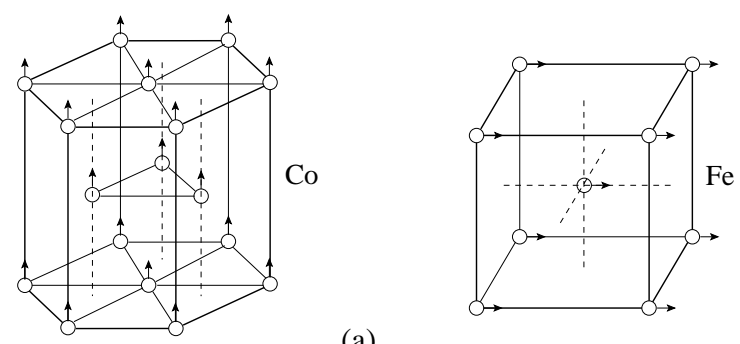

(a)
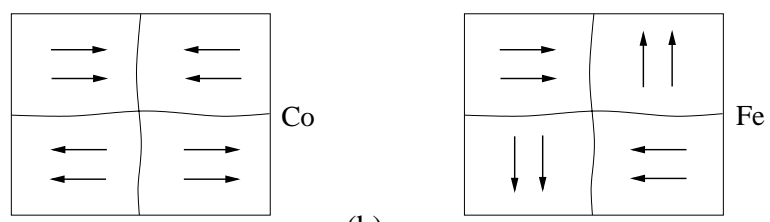

(b)

Figure 1: (a) Crystallographic orientation of magnetic moments in cobalt and iron (after [4]). (b) Corresponding domain structure which reflects the uniaxial or hexagonal anisotropy of cobalt and the cubic anisotropy of iron.

this will be at least approximately true for materials having uniaxial crystalline anisotropies or systems in which uniaxial stresses dominate the crystalline structure. Materials exhibiting uniaxial crystalline anisotropies include cobalt and a number of rare earth metals and alloys (e.g., Terbium single crystals). This produces domain structures in which moments are highly parallel or antiparallel as depicted in Figure 1.

To illustrate a regime in which stresses dominate crystalline anisotropies, consider the TerfenolD transducer depicted in Figure 2(a) and detailed in [45-47]. In present manufacturing processes, Terfenol-D crystals are grown in Dendrite sheets oriented in the $[11 \overline{2}]$ directions as depicted in Figure 2(b). At the prestress levels employed in present transducer designs, the preferred orientation of domains is shifted from the original eight $\langle 111\rangle$ magnetic easy axes to the two axes [111] and [111] perpendicular to the [112] $]$ axis of the rod. In the presence of a field $H$ generated by an applied current $I$ to the solenoid, moments first rotate irreversibly to the [111] easy axis and then rotate reversibly to the $[11 \overline{2}]$ axis. For these transducer constructions, stress anisotropies can dominate crystalline anisotropies to provide regimes for which the assumption of two spin orientations provides reasonable approximations.
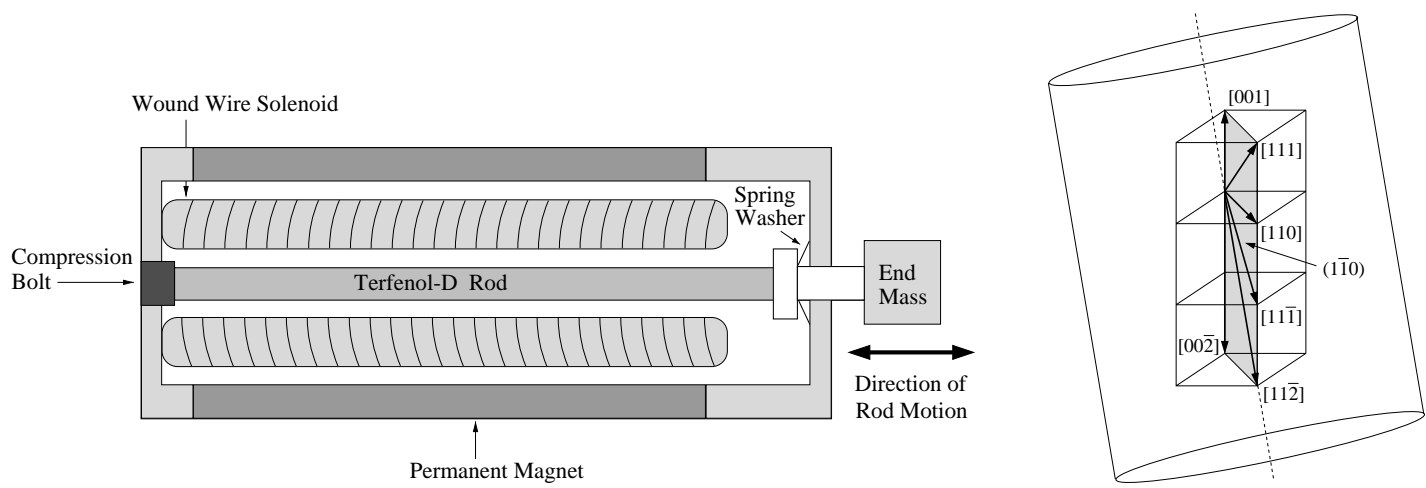

Figure 2: (a) Cross section of a typical Terfenol-D magnetostrictive transducer. (b) Orientation of Terfenol-D crystals. 
Finally, this assumption can be motivated by noting that from a quantum perspective, spins cannot be uniformly oriented and one allowed orientation is parallel and opposite to an applied field. This interpretation can be used to explain the accuracy of the theory for quantifying the behavior of certain materials such as iron which has the crystalline and domain properties illustrated in Figure 1 and exhibits cubic anisotropy.

\subsubsection{Temperature-Dependent Helmholtz Energy}

The statistical mechanics model is based on an approximation to the Ising model first proposed by Gorsky in the analysis of order-disorder transitions in binary alloys [48]. In this context, it was later extended by Bragg and Williams to include the concept of long range order [49-51]. An underlying tenet in the Bragg-Williams theory, which simplifies subsequent computations, is the assumption that the energy of an individual atom is determined by the average order of the system rather than the fluctuating states of adjacent atoms. For this reason, the model is often termed the mean field or molecular field approximation to the Ising model. To construct a ferromagnetic model, we make the same assumption regarding magnetic moments or spins. Further details about this approach, including some discussion concerning its application to ferromagnetic materials, can be found in $[52,53]$.

We consider an arbitrary lattice of volume $V$ and mass $\nu$ comprised of $N=N_{+}+N_{-}$cells, each of which is assumed to contain one spin or magnetic moment. In accordance with the Ising assumptions, the spin orientations are constrained to be $\sigma_{i}= \pm 1$, and $N_{+}$and $N_{-}$respectively denote the number of positive and negative spins in the lattice. We note that due to the initial assumption of material homogeneity, this lattice structure is representative of that found throughout the structure. If each spin has a moment $m$, the magnetization for the lattice is

$$
\begin{aligned}
M & =\frac{m}{V} \sum_{i=1}^{N} \sigma_{i} \\
& =\frac{M_{s}}{N}\left(N_{+}-N_{-}\right),
\end{aligned}
$$

from which it follows that

$$
N_{+}=\frac{N}{2}\left(1+\frac{M}{M_{s}}\right), \quad N_{-}=\frac{N}{2}\left(1-\frac{M}{M_{s}}\right) .
$$

Here $M_{s}=N m / V$ denotes the technical saturation magnetization which occurs when all moments are aligned. Additionally, we make the assumption that only adjacent moments interact.

To quantify the energy required to reorient moments, we employ the mean field approximation of Bragg and Williams and make the assumption that the average exchange energy $\Phi$ is proportional to $M / M_{s}$; that is,

$$
\Phi=\Phi_{0} M / M_{s}
$$

where $\Phi_{0}$ denotes the energy required to reorient a single moment if the lattice is completely ordered $\left(M=M_{s}\right)$. For the case of a homogeneous lattice, $\Phi_{0}$ is considered to be constant. For nonhomogeneous and polycrystalline materials, $\Phi_{0}$ will be considered as a manifestation of an underlying statistical distribution as discussed in Section 4. We also note that $\Phi_{0}$ is related to the exchange integral $\mathcal{J}$ employed in (6) through the expression

$$
\Phi_{0}=2 \xi \mathcal{J}
$$


where $\xi$ denotes the number of neighbors adjacent to a site. Hence $\xi=2$ for a 1-D lattice chain, $\xi=4$ for a 2 -D rectangular lattice, and $\xi=6,8$ or 12 , respectively, for 3 -D cubic, body-centered cubic, or face-centered cubic lattices. The fact that (11) is independent of the exact lattice structure has led Pathria to refer to the subsequent model as a zeroth approximation of the Ising model [53].

We now consider the decrease in internal energy due to a change from $N_{+}$to $N_{+}+d N_{+}$. From the mean field approximation (11), each switch requires $\Phi_{0} \frac{M}{M_{s}}$ in energy so the change in internal energy for a unit volume is

$$
\begin{aligned}
d U_{E} & =-\frac{\Phi_{0} M}{V M_{s}} d N_{+} \\
& =-\frac{\phi_{0} M}{V M_{s}} \cdot \frac{N}{2 M s} d M
\end{aligned}
$$

where the second equality follows from (10). Integration, in combination with (10), yields the relation

$$
U_{E}=\frac{\Phi_{0} N}{4 V}\left(1-\frac{M^{2}}{M_{s}^{2}}\right)+U_{0}
$$

for the exchange energy. Since we are interested in relative rather than absolute measures of energy, we take $U_{0}=0$ which specifies that the completely ordered state has an internal energy of zero.

As detailed in [52], the entropy $S$ for the system is given by

$$
S=\frac{k N}{V} \ln W
$$

where $k$ is Boltzmann's constant and $W$ quantifies the number of ways moments can be arranged in the lattice to yield the magnetization $M$. By noting that this is equivalent to arranging $N_{+}$moments in $N$ sites, and employing Stirling's approximation

$$
\ln x !=x \ln x-x,
$$

the entropy can be formulated as

$$
\begin{aligned}
S & =\frac{k}{V} \ln \left[\left(\begin{array}{c}
N \\
N_{+}
\end{array}\right)\right] \\
& =\frac{k}{V} \ln \left[\frac{N !}{N_{-} ! N_{+} !}\right] \\
& =\frac{k N}{V}\left[\ln 2-\frac{1+M / M_{s}}{2} \ln \left(1+\frac{M}{M_{s}}\right)-\frac{1-M / M_{s}}{2} \ln \left(1-\frac{M}{M_{s}}\right)\right]+S_{0} \\
& =\frac{-k N}{2 V M_{s}}\left[M \ln \left(\frac{M+M_{s}}{M_{s}-M}\right)+M_{s} \ln \left(1-\left(\frac{M}{M_{s}}\right)^{2}\right)\right]+S_{0}
\end{aligned}
$$

where $S_{0}=\frac{k N}{V} \ln 2$.

The Helmholz energy for the lattice is then

$$
\begin{aligned}
\psi(M, T) & =U-S T \\
& =\frac{\Phi_{0} N}{4 V}\left[1-\left(M / M_{s}\right)^{2}\right]+\frac{T k N}{2 V M_{s}}\left[M \ln \left(\frac{M+M_{s}}{M_{s}-M}\right)+M_{s} \ln \left(1-\left(M / M_{s}\right)^{2}\right)\right] \\
& =\frac{H_{h} M_{s}}{2}\left[1-\left(M / M_{s}\right)^{2}\right]+\frac{H_{h} T}{2 T_{c}}\left[M \ln \left(\frac{M+M_{s}}{M_{s}-M}\right)+M_{s} \ln \left(1-\left(M / M_{s}\right)^{2}\right)\right]
\end{aligned}
$$




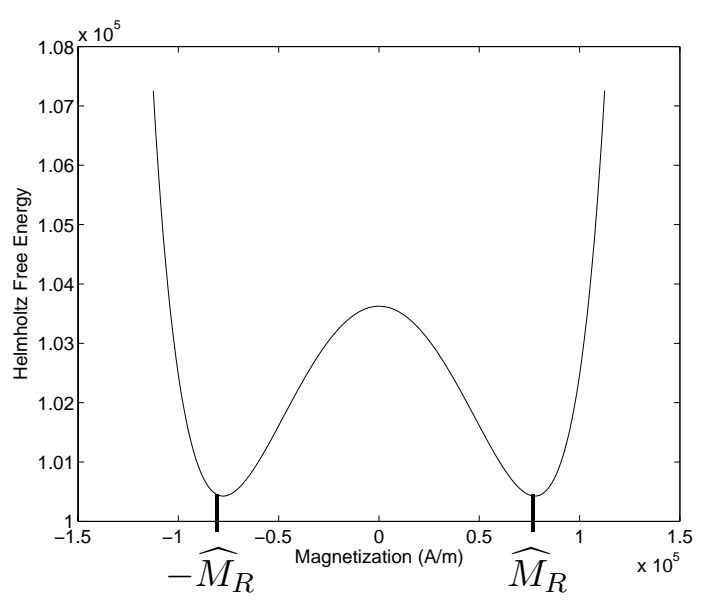

(a)

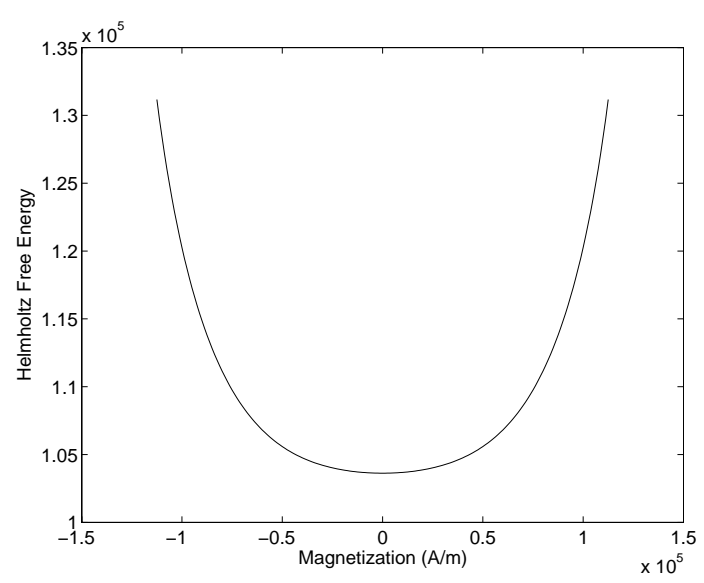

(b)

Figure 3: Helmholtz energy specified by (18) for (a) $T<T_{c}$, and (b) $T>T_{c}$.

where $H_{h}=\frac{N \Phi_{0}}{2 V M_{s}}$ is a bias field and $T_{c}=\frac{\Phi_{0}}{2 k}$ denotes the Curie temperature. The initial assumption that the exchange energy $\Phi_{0}$ is constant implies that $H_{h}$ will also be constant for homogeneous materials. This assumption will be relaxed in Section 4 to include statistically distributed values of $H_{h}$ for modeling nonhomogeneous and polycrystalline materials.

As illustrated in Figure 3, the Helmholtz relation (18) exhibits double well behavior for temperatures $T<T_{c}$ and single well behavior for $T \geq T_{c}$. This is consistent with the transition exhibited between ferromagnetic and paramagnetic phases.

\subsubsection{Temperature-Invariant Helmholtz Energy}

Whereas the Helmholtz relation (18) incorporates a number of the properties desired for microscopic material characterization, the logarithmic components add complexity to resulting macroscopic models which can reduce the efficiency of algorithms when considered for real-time implementation. For applications requiring high efficiency, a simplified Helmholtz relation can be obtained by retaining the quadratic behavior of (18) for fixed temperature regimes.

To determine an appropriate piecewise quadratic model, we consider the Taylor expansion

$$
\psi(M, T)=\psi\left(M_{0}, T\right)+\left(M-M_{0}\right) \frac{\partial \psi}{\partial M}\left(M_{0}, T\right)+\frac{\left(M-M_{0}\right)^{2}}{2} \frac{\partial^{2} \psi}{\partial M^{2}}\left(M_{0}, T\right)+\mathcal{O}\left(M^{3}\right)
$$

for fixed $T<T_{c}$, where $M_{0}$ is taken to be an equilibrium, and

$$
\begin{gathered}
\frac{\partial \psi}{\partial M}=\frac{-H_{h}}{M_{s}} M+\frac{H_{h} T}{T_{c}} \tanh ^{-1}\left(M / M_{s}\right), \\
\frac{\partial^{2} \psi}{\partial M^{2}}=\frac{-H_{h}}{M_{s}}+\frac{H_{h} T}{T_{c} M_{s}\left[1-\left(M / M_{s}\right)^{2}\right]} .
\end{gathered}
$$

From the necessary condition $\frac{\partial \psi}{\partial M}\left(M_{0}, T\right)=0$, the equilibria are determined to be the two stable solutions to

$$
M=M_{s} \tanh \left(\frac{\alpha M}{a(T)}\right)
$$


in addition to the unstable solution $M=0$. The parameters $\alpha$ and $a(T)$ are specified by

$$
\alpha=\frac{H_{h}}{M_{s}} \quad, \quad a(T)=\frac{H_{h} T}{T_{c}} .
$$

If we let $\widehat{M}_{R}$ and $-\widehat{M}_{R}$ denote locations of the stable equilibria determined through solution of (21), as depicted in Figure 3(a), then it can be directly established that the quadratic approximations to (19) in neighborhoods of the equilibria $M_{0}=0,-\widehat{M}_{R}$ and $\widehat{M}_{R}$ are

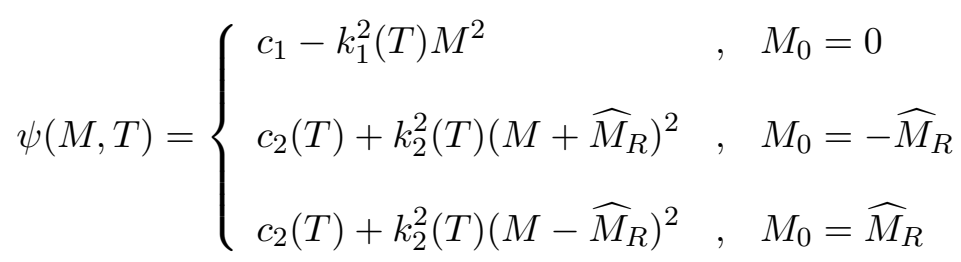

where

$$
c_{1}=\frac{H_{h} M_{s}}{2} \quad, \quad k_{1}^{2}(T)=\frac{H_{h}}{2 M_{s} T_{c}}\left(T_{c}-T\right)
$$

with analogous, but more complicated, expressions for $c_{2}(T)$ and $k_{2}(T)$.

For fixed temperature regimes, this motivates the consideration of the piecewise quadratic definition

$$
\psi(M)= \begin{cases}\frac{1}{2} \eta\left(M+M_{R}\right)^{2} & , M \leq-M_{I} \\ \frac{1}{2} \eta\left(M-M_{R}\right)^{2} & , M \geq M_{I} \\ \frac{1}{2} \eta\left(M_{I}-M_{R}\right)\left(\frac{M^{2}}{M_{I}}-M_{R}\right) & ,|M|<M_{I}\end{cases}
$$

as a second choice for the Helmholtz energy. As illustrated in Figure 4(a), $M_{I}$ and $M_{R}$ respectively denote the inflection point and magnetization at which the minimum of $\psi$ occurs. It will be established in subsequent discussion that $M_{I}$ and $M_{R}$ represent parameters to be estimated through a least squares fit to data when quantifying specific materials.
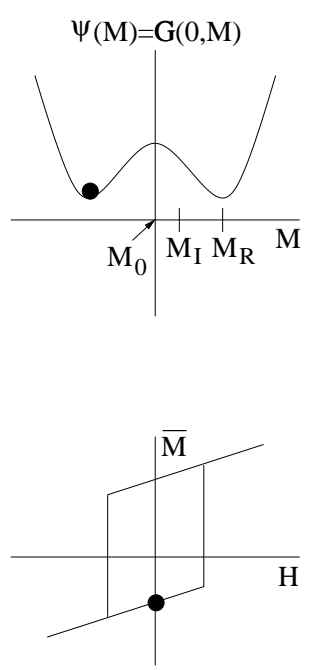

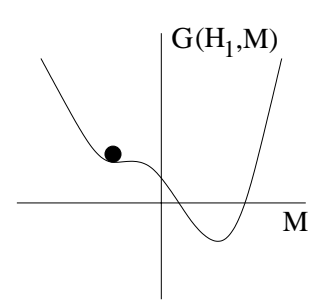

(a)

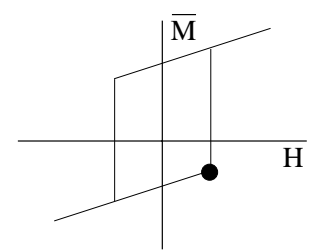

(b)
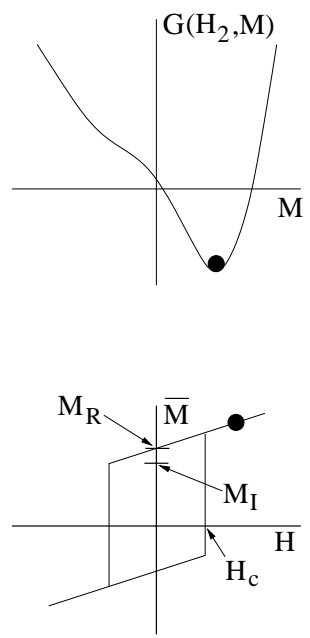

Figure 4: (a) Helmholtz energy $\psi$ and Gibbs energy $G$ for increasing field $H\left(H_{2}>H_{1}>0\right)$. (b) Dependence of the local average magnetization $\bar{M}$ on the field in the absence of thermal activation. 


\subsection{Gibbs Energy}

The Helmholtz relations (18) or (25) quantify certain aspects of the exchange energy $U_{E}$ for ferromagnetic materials. To incorporate the work done by an applied field, we note from (8) that the magnetostatic energy can be expressed as $U_{M}=\mu_{0} \mathbf{m} \cdot \mathbf{H}$, where $\mu_{0}$ denotes the magnetic permeability, and form the Gibbs energy relations

$$
G(H, M, T)=\psi(M, T)-\mu_{0} H M
$$

or

$$
G(H, M, T)=\psi(M, T)-H M
$$

by incorporating $\mu_{0}$ into $\psi$. For increasing $H$, the behavior of $G$ with $\psi$ given by (25) is depicted in Figure 4(a). In the absence of anisotropic effects or applied stresses, $G$ approximates the energy landscape exhibited at the lattice level in homogeneous materials.

\section{Local Average and Anhysteretic Magnetizations}

\subsection{Local Magnetization}

For conditions in which thermal after-effects [2] are negligible, the local average magnetization $\bar{M}$ at the lattice level is determined by minimizing the Gibbs relations (26) or (27) whereas the Gibbs energy must be balanced with the thermal energy through Boltzmann principles if thermal effects are significant. We consider these two regimes in Sections 3.1.1 and 3.1.2 and then illustrate in Section 3.1.3 that the model which incorporates thermal energy limits to the case of no thermal activation when reference volumes $V$ are taken to be arbitrarily large.

\subsubsection{Negligible Thermal Effects}

For conditions in which thermal after-effects are negligible, the local average magnetization $\bar{M}$ is determined from the necessary conditions

$$
\frac{\partial G}{\partial M}=0, \quad \frac{\partial^{2} G}{\partial M^{2}}>0 .
$$

When the statistical mechanics relation (18) is employed for the Helmholtz energy, this yields the Ising relation

$$
\bar{M}(H)=M_{s} \tanh \left(\frac{H+\alpha M}{a(T)}\right)
$$

where $\alpha$ and $a(T)$ are defined in (22). The behavior of the kernel or hysteron is illustrated in Figure 5(a).

Remark 1. The Ising relation (29), whose input is the effective field

$$
H_{e}=H+\alpha M
$$

is fundamental in a number of hysteresis models for ferromagnetic and ferroelectric compounds. This relation was directly employed for quantifying the anhysteretic component of unified models developed in [54]. Furthermore, it is illustrated in [54,55] that if one relaxes the constraint that moments have only the orientations $\sigma_{i}= \pm 1$ and considers uniformly distributed moments, one obtains the Langevin relation $M=\mathcal{L}\left(H_{e}\right) \equiv M_{s}\left[\operatorname{coth}\left(H_{e} / a\right)-a / H_{e}\right]$ which agrees with the Ising 


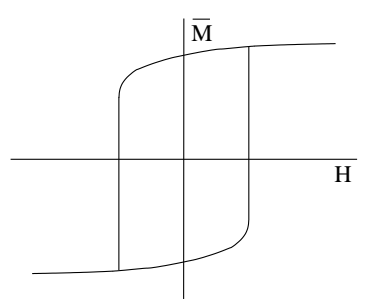

(a)

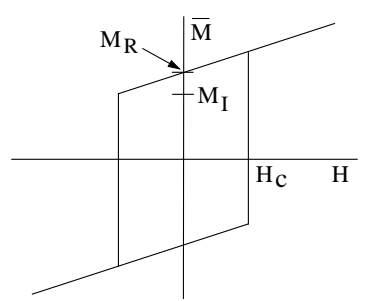

(b)

Figure 5: (a) Limiting kernel (29) obtained with the Helmholtz energy (18) in the absence of thermal activation. (b) Limiting kernel (32) provided by the Helmholtz relation (25).

relation $M=M_{s} \tanh \left(H_{e} / a\right)$ through first order terms - see [2, 4] for a derivation of the Langevin equation in the context of magnetic materials. The Langevin model $M=M_{s} \mathcal{L}\left(H_{e}\right)$, with $H_{e}$ specified by (30), is employed when quantifying the anhysteretic magnetization in the domain wall theory of Jiles and Atherton [10,11] as well as the transducer models based on that theory [45-47] - e.g., see (1). Finally, translates of the Ising relation $r(x)=\tanh (x)$ form appropriate ridge functions for generalized Preisach, or Krasnosel'skiü-Pokrovskǐ characterizations [56-58]. Hence this relation plays a fundamental role in two of the present macroscopic theories outlined in Section 1.

The local average magnetization $\bar{M}$ resulting from (25) is elementary in the sense that it is piecewise linear but is complicated by the fact that a history of moment switches must be maintained to ascertain which branch of the hysteron is active. Enforcement of the necessary condition(28) yields

$$
\bar{M}=\frac{1}{\eta} H+M_{R} \delta
$$

where $\delta=1$ for positively oriented moments and $\delta=-1$ for negative orientations. To quantify $\delta$ in terms of initial moment configurations and previous switches, we employ Preisach notation e.g., see $[33,56,58]$ — and take

$$
\left[\bar{M}\left(H ; H_{c}, \xi\right)\right](t)= \begin{cases}{\left[\bar{M}\left(H ; H_{c}, \xi\right)\right](0)} & , \tau(t)=\emptyset \\ \frac{H}{\eta}-M_{R} & , \tau(t) \neq \emptyset \text { and } H(\max \tau(t))=-H_{c} \\ \frac{H}{\eta}+M_{R} & , \tau(t) \neq \emptyset \text { and } H(\max \tau(t))=H_{c} .\end{cases}
$$

Here

$$
\left[\bar{M}\left(H ; H_{c}, \xi\right)\right](0)= \begin{cases}\frac{H}{\eta}-M_{R} & , H(0) \leq-H_{c} \\ \xi & ,-H_{c}<H(0)<H_{c} \\ \frac{H}{\eta}+M_{R} & , H(0) \geq H_{c}\end{cases}
$$

denotes the initial moment distribution and transition times are designated by

$$
\tau(t)=\left\{t \in\left(0, t_{f}\right] \mid H(t)=-H_{c} \text { or } H(t)=H_{c}\right\}
$$

where $t_{f}$ denotes the final time under consideration.

The dependence of $\bar{M}$ on the local coercive field

$$
H_{c}=\eta\left(M_{R}-M_{I}\right)
$$

is indicated as a prelude to the discussion in Section 4 where $H_{c}$ is assumed distributed to accommodate material nonhomogeneities. 
The behavior of the limiting kernel (31) or (32) is compared with its statistical mechanics counterpart (29) in Figure 5. It is observed that the primary difference between the kernels occurs in the saturation behavior at high fields. The kernel (32) predicts a linear relation between $H$ and $\bar{M}$ after moment switching whereas the kernel (29) exhibits saturation behavior to a local average magnetization value $M_{s}$.

Remark 2. A comparison of the upper and lower branches of the Ising kernel plotted in Figure 5(a) illustrates that this kernel, obtained from the energy relation (18), yields noncongruent behavior. Hence it can be used to characterize the noncongruency measured in certain operating regimes.

Remark 3. The limiting kernel (32) provides reversible behavior at high fields due to the fact that the kernel does not saturate.

\subsubsection{Thermal After-Effects}

To incorporate the thermal mechanisms which produce phenomena such as after-effects [2], it is necessary to balance the Gibbs energy $G$ with the relative thermal energy $k T / V$, over the reference volume $V$, through the Boltzmann density relation

$$
\mu(G)=C e^{-G V / k T}
$$

which specifies the probability of attaining an energy level $G$ for a fixed field input. The constant $C$ is chosen to ensure a probability of unity when $\mu$ is integrated over all admissible moment configurations.

\section{Gaussian Behavior of $\mu$}

To illustrate the behavior of $\mu$ for the Gibbs energy $G=\psi-H M$ constructed using the piecewise Helmholtz model (25), we consider the specific energy profile depicted in Figure 6 for which it is assumed that $H>0$ and $G\left(M_{m i n}^{+}\right)<G\left(M_{m i n}^{-}\right) \leq G\left(M_{0}\right)$. The relative minima

$$
\begin{aligned}
& M_{\text {min }}^{-}(H)=\frac{H}{\eta}-M_{R}, \\
& M_{\text {min }}^{+}(H)=\frac{H}{\eta}+M_{R}
\end{aligned}
$$

result from the necessary condition (28) utilized when constructing the limiting model (31) or (32). The local coercive field $H_{c}$ for which $M_{\text {min }}^{-}=-M_{I}=M_{0}$ is given by (35).

From (25), it follows that for $M<-M_{I}$, the Boltzmann probability can be formulated as

$$
\begin{aligned}
\mu(G(H, M)) & =C(T) e^{-G(H, M) V / k T} \\
& =\frac{e^{-\left[\frac{1}{2} \eta\left(M+M_{R}\right)^{2}-H M\right] V / k T}}{\int_{-\infty}^{-M_{I}} e^{-\left[\frac{1}{2} \eta\left(M+M_{R}\right)^{2}-H M\right] V / k T} d M} \\
& =\frac{e^{-\left(M-M_{\text {min }}^{-}\right)^{2} \eta V / 2 k T}}{\int_{-\infty}^{-M_{I}} e^{-\left(M-M_{\text {min }}^{-}\right)^{2} \eta V / 2 k T} d M} \\
& =\bar{C}(T, \beta) e^{-\left(M-M_{\text {min }}^{-}\right)^{2} / 2 \beta^{2}}
\end{aligned}
$$




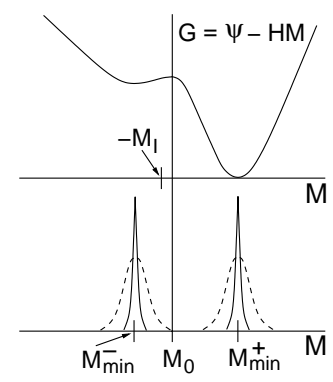

(a)

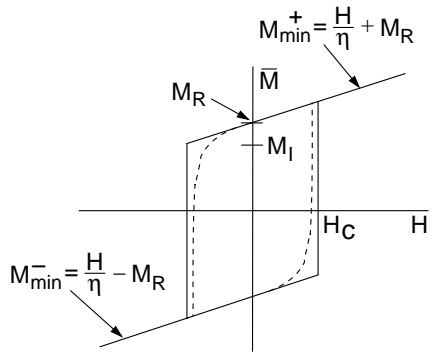

(b)

Figure 6: (a) Gibbs energy profile with a high level (-- ) and low level ( - ) of thermal activation in the Boltzmann probability $\mu(G)=C e^{-G V / k T}$. (b) Local magnetization $\bar{M}$ given by equation (49) with high thermal activation $(---)$ and limiting magnetization $\bar{M}$ specified by (32) in the absence of thermal activation (-).

where

$$
\begin{aligned}
& \beta=\sqrt{\frac{k T}{\eta V}}, \\
& \bar{C}(T, \beta)=\left[\int_{-\infty}^{-M_{I}} e^{-\left(M-M_{m i n}^{-}\right)^{2} / 2 \beta^{2}(T)} d M\right]^{-1} .
\end{aligned}
$$

Similarly, for $M>M_{I}$, the probability has the form

$$
\mu(M)=\frac{e^{-\left(M-M_{m i n}^{+}\right)^{2} \eta V / 2 k T}}{\int_{-\infty}^{-M_{I}} e^{-\left(M-M_{m i n}^{+}\right)^{2} \eta V / 2 k T} d M} .
$$

Relations (38) and (40) illustrate the Gaussian behavior of the Boltzmann probabilities for the piecewise quadratic Helmholtz function $\psi$ while (39) illustrates that the variance $\beta^{2}$ is proportional to the relative thermal energy $k T / V$. From a physical perspective, low relative thermal energy implies that fewer moments achieve the energy required to overcome energy barriers thus producing steep transitions in the local relation between $H$ and $\bar{M}$.

To illustrate the Dirac nature of $\mu(G)$ in (38) as $k T / V$ decreases, let $j=1 / \beta$ and define the sequence

$$
\phi_{j}\left(M-M_{\text {min }}^{-}\right)=\left\{\begin{array}{cc}
\bar{C}(T, j) e^{-\left(M-M_{\text {min }}^{-}\right)^{2} j^{2} / 2} & , \quad M \leq-M_{I} \\
0 & , \quad M>-M_{I} .
\end{array}\right.
$$

The sequence $\left\{\phi_{j}\right\}$ satisfies properties (i)-(iii) of Theorem 1 in 7 and hence constitutes a Dirac family. It follows immediately that

$$
\begin{aligned}
\lim _{k T / v \rightarrow 0} \mu(M) & =\lim _{j \rightarrow \infty} \phi_{j}(M) \\
& =\delta\left(M-M_{\text {min }}^{-}\right) .
\end{aligned}
$$

Analogous behavior is exhibited at $M_{\text {min }}^{+}$as depicted in Figure 6(b).

\section{Transition Likelihoods and Local Average Magnetization}

Because the Boltzmann relation (36) quantifies the balance between the Gibbs and relative thermal energies, it is employed when modeling the fraction of positively and negatively oriented moments, the average magnetizations due to the two configurations, and the likelihoods that moments changes configurations for a given input field level. 
Recalling that $N_{-}$and $N_{+}$respectively denote the number of negatively and positively oriented moments, we denote the respective moment fractions by $x_{-}=N_{-} / N$ and $x_{+}=N_{+} / N$ where

$$
x_{-}+x_{+}=1
$$

since $N_{-}+N_{+}=1$. The evolution of moment fractions is determined by the differential equations

$$
\begin{aligned}
& \dot{x}_{+}=-p_{+-} x_{+}+p_{-+} x_{-} \\
& \dot{x}_{-}=-p_{-+} x_{-}+p_{+-} x_{+}
\end{aligned}
$$

which can be simplified to

$$
\dot{x}_{+}=-p_{+-} x_{+}+p_{-+}\left(1-x_{+}\right)
$$

through the identity (43). For a demagnetized material, initial conditions can be taken to be $\widehat{x}_{-}=$ $\widehat{x}_{+}=1 / 2$.

The expected or average magnetizations due to negatively and positively oriented moments are defined by

$$
\left\langle M_{-}\right\rangle=\int_{-\infty}^{M_{0}(T)} M \mu(M) d M \quad, \quad\left\langle M_{+}\right\rangle=\int_{M_{0}(T)}^{\infty} M \mu(M) d M
$$

where $M_{0}(T)$ denotes the unstable equilibrium of $G$ as depicted in Figure 6(a). For the piecewise quadratic Helmholtz energy functional (25), the evaluation of the integrals in (46) is simplified by replacing the limit $M_{0}(T)$ respectively by $-M_{I}$ and $M_{I}$ in the definitions of $\left\langle M_{-}\right\rangle$and $\left\langle M_{+}\right\rangle$. This can be motivated by observing that maximum restoring forces occur at the inflection points as detailed on pages 332-333 of [3] or pages 486-487 of [2]. Furthermore, these points coincide in the limit of negligible thermal activation as illustrated in Section 3.1.3. With this approximation, we have

$$
\left\langle M_{-}\right\rangle=\frac{\int_{-\infty}^{-M_{I}} M e^{-G(H, M) V / k T} d M}{\int_{-\infty}^{-M_{I}} e^{-G(H, M) V / k T} d M}, \quad\left\langle M_{+}\right\rangle=\frac{\int_{M_{I}}^{\infty} M e^{-G(H, M, T) V / k T} d M}{\int_{M_{I}}^{\infty} e^{-G(H, M, T) V / k T} d M} .
$$

The likelihood of switching from a positive moment orientation to negative, and conversely, are respectively quantified by

$$
p_{+-}=\frac{1}{\mathcal{T}(T)} \frac{\int_{M_{I}-\epsilon}^{M_{I}} e^{-G(E, M) V / k T} d M}{\int_{M_{I}-\epsilon}^{\infty} e^{-G(E, M) V / k T} d M} \quad, \quad p_{-+}=\frac{1}{\mathcal{T}(T)} \frac{\int_{-M_{I}}^{-M_{I}+\epsilon} e^{-G(E, M) V / k T} d M}{\int_{-\infty}^{-M_{I}+\epsilon} e^{-G(E, M) V / k T} d M}
$$

where $\epsilon$ is taken to be a small positive constant. The quotient of integrals is a probability and hence is unitless. The relaxation time $\mathcal{T}$ is the reciprocal of the frequency at which moments attempt to switch so $\frac{1}{\mathcal{T}}$ has units of $\frac{1}{\sec }$. This yields the correct units in the differential equations (44) and (45). Moreover, we note that $\mathcal{T}^{2}$ is considered to be inversely proportional to the relative thermal energy so that $\mathcal{T}(T)=\mathcal{T}_{1} \sqrt{V / k T}$; hence increased temperature lead to increased thermal relaxation behavior. For materials having a single relaxation time, $\mathcal{T}_{1}$ is constant whereas variable relaxation times may need to be identified for materials exhibiting distributed relaxation behavior.

With the moment fractions, expected magnetization values, and transition likelihoods thus defined, the local average magnetization for the lattice is

$$
\bar{M}=x_{+}\left\langle M_{+}\right\rangle+x_{-}\left\langle M_{-}\right\rangle .
$$


The behavior of the local model (49), which incorporates thermal after-effects (thermal relaxation) is compared in Figure 6(b) with the relation (32) obtained by simply minimizing the Gibbs energy. For values of $k T / V$ on the order of $G$, a significant number of moments achieve the relative thermal energy required for switching in advance of the local coercive field $H_{c}$. This produces a smooth transition between the limiting minima (37) of the hysteron. For diminishing values of $k T / V$ as compared with $G$, fewer moments achive the thermal energy required for pre-coercive switching which produces increasingly steep transitions between orientations. This convergence is rigorously established in the next section.

\subsubsection{Limiting Behavior of Local Magnetization Model}

The local model (49) incorporates thermal after-effects by employing the Boltzmann relation (36) to balance the Gibbs energy $G$ and relative thermal energy $k T / V$ whereas the local model (32) was derived in the absence of thermal relaxation mechanisms simply by minimizing the Gibbs energy. We rigorously establish here the convergence of (49) to (32) in the limit $k T / V \rightarrow 0$ of increasing control volumes and hence diminishing relative thermal energies. To clarify the discussion, we consider the representative energy landscape depicted in Figure 6(a) - however, the analysis techniques accommodate general energy configurations.

We consider first the convergence of the expected magnetization relations (47). For negative moments, we consider the Dirac sequence $\left\{\phi_{j}\right\}$ defined in (41), define the function $f(M)=M$, and consider the interval $[a, b]=\left[M_{\text {min }}^{-}, M_{0}\right]$ where $M_{\text {min }}^{-}$is defined in (37). Hence $f$ is continuous on $\mathbb{R}$ and satisfies the decay property (iv) in Theorem 1 of 7 . It then follows that

$$
\begin{aligned}
\lim _{k T / V \rightarrow 0}\left\langle M_{-}\right\rangle & =\lim _{j \rightarrow \infty} \int_{-\infty}^{\infty} M \phi_{j}\left(M-M_{\text {min }}^{-}\right) d M \\
& =M_{\text {min }}^{-} .
\end{aligned}
$$

Analogous arguments can be used to demonstrate that $\left\langle M_{+}\right\rangle \rightarrow M_{\min }^{+}$as $k T / V \rightarrow 0$.

To illustrate the convergence of the transition likelihoods for a fixed relaxation time $\mathcal{T}(T)$, we modify the sequence $\left\{\phi_{j}\right\}$ defined in (41) for the interval $\left(-\infty,-M_{I}+\epsilon\right]$. The function $f$ is specified to be

$$
f(M)= \begin{cases}0, & M<-M_{I} \\ 1, & M \geq-M_{I}\end{cases}
$$

and the interval $[a, b]$ is taken to be $\left[-2 M_{m i n}^{-},-M_{I}\right]$ or $\left[-M_{I}, M_{0}\right]$. Since $f$ again satisfies (iv) in Theorem 1 of 7 , we obtain the convergence

$$
\begin{aligned}
\lim _{k T / V \rightarrow 0} p_{-+} & =\lim _{j \rightarrow \infty} \frac{1}{\mathcal{T}(T)} \int_{-\infty}^{\infty} f(M) \phi_{j}\left(M-M_{m i n}^{-}\right) d M \\
& =\frac{1}{\mathcal{T}(T)} \begin{cases}0 & , \quad H<H_{c} \\
1, & H \geq H_{c}\end{cases}
\end{aligned}
$$

for $H_{c}$ defined by (35). Similar analysis for positively oriented moments in the considered energy landscape yields

$$
\lim _{k T / V \rightarrow 0} p_{+-}=0 .
$$

We let $\zeta_{+}=x_{+}(0)$ and $\zeta_{-}=x_{-}(0), \zeta_{+}+\zeta_{-}=1$, denote the initial moment fractions and consider the behavior of the differential equation (45) governing the evolution of $x_{+}$. Under the assumption 
that $H$, which is parameterized with respect to time, is increasing, we let $t=t_{c}$ denote the time at which $H(t)=H_{c}$. The solution to (45) in the limit $k T / V \rightarrow 0$ is then

$$
x_{+}(t)= \begin{cases}\zeta_{+} & , t<t_{c} \\ 1-\left(1-\zeta_{+}\right) e^{-\left(t-t_{c}\right) / \mathcal{T}} & , \quad t \geq t_{c}\end{cases}
$$

and the limiting local magnetization is

$$
[\bar{M}(H)](t)= \begin{cases}\frac{H(t)}{\eta}+\left(2 \zeta_{+}-1\right) M_{R} & , t<t_{c} \\ \frac{H(t)}{\eta}+\left[1-2\left(1-\zeta_{+}\right) e^{-\left(t-t_{c}\right) / \mathcal{T}}\right] M_{R} & , \quad t \geq t_{c} .\end{cases}
$$

For large $t$ or small $\mathcal{T}, \bar{M}(H)$ limits to

$$
\begin{aligned}
{[\bar{M}(H)](t) } & = \begin{cases}\frac{H(t)}{\eta}+\left(2 \zeta_{+}-1\right) M_{R}, t<t_{c} \\
\frac{H(t)}{\eta}+M_{R}, t \geq t_{c}\end{cases} \\
& = \begin{cases}\zeta_{-}\left[M_{\text {min }}^{-}(H)\right](t)+\zeta_{+}\left[M_{\text {min }}^{+}(H)\right](t) & , t<t_{c} \\
{\left[M_{\text {min }}^{+}(H)\right](t)} & , t \geq t_{c}\end{cases}
\end{aligned}
$$

which is precisely (32).

Remark 4. To summarize, the linear kernel (31) or (32) can be accurately employed when thermal effects are negligible $(k T / V$ is small) and relaxation times $\mathcal{T}$ are small compared with drive frequencies. Otherwise, one should employ the kernel (49) or an asymptotic relation of the form (55) to accommodate thermal activation or long relaxation times.

\subsection{Local Anhysteretic Magnetization}

The relations (32) and (49) characterize the local hysteretic $H$ - $M$ behavior at the lattice level when the piecewise quadratic relation (25) is used to quantify the Helmholtz energy. The energy framework used to establish these relations also quantifies the anhysteretic $H-M$ behavior which is experimentally achieved by applying sufficiently large AC fields superimposed on a DC bias field. From a theoretical perspective, the local anhysteretic magnetization $\bar{M}_{a n}$ represents the locus of magnetization values which would occur in materials devoid of inclusions. It can also be theoretically formulated as the magnetization achieved when relaxation times $\mathcal{T}(T)$ are sufficiently small compared with drive frequencies that moments achieve global equilibria.

We illustrate the latter theoretical interpration in the context of the local magnetization model (49) derived under the assumption that $G$ and $k T / V$ are balanced through the relation (36). The condition of moment equilibrium yields $\dot{x}_{+}=\dot{x}_{-}=0$ in (44) which in turn implies that equilibrium solutions $\bar{x}_{+}$and $\bar{x}_{-}$satisfy the relation

$$
\frac{\bar{x}_{+}}{\bar{x}_{-}}=\frac{p_{-+}}{p_{-+}} .
$$

To demonstrate the implication of (57), consider first the case when $H=0$. From the definition (48), it follows immediately that $p_{-+}=p_{+-}$and hence $\bar{x}_{+}=\bar{x}_{-}$. From the conservation relation $\bar{x}_{+}+\bar{x}_{-}=1$, it is deduced that $\bar{x}_{+}=\bar{x}_{-}=\frac{1}{2}$, regardless of the initial conditions $x_{+}(0)$ and $x_{-}(0)$. The rate at which the relations converge to equilibrium values is determined by the relaxation time $\mathcal{T}(T)$, with smaller values of $\mathcal{T}$ producing more rapid equilibration. 
To ascertain the resulting anhysteretic magnetization for $H=0$, we note that the symmetry of (47) implies that

$$
\left\langle\bar{M}_{+}\right\rangle=-\left\langle\bar{M}_{-}\right\rangle
$$

at equilibrium. When combined with the fact that $\bar{x}_{+}=\bar{x}_{-}=\frac{1}{2},(49)$ yields

$$
\bar{M}_{a n}(H=0)=0 .
$$

The field-dependence of $p_{+-}, p_{-+},\left\langle M_{+}\right\rangle$and $\left\langle M_{-}\right\rangle$precludes a similar exploitation of symmetries for $H \neq 0$. However, $\bar{M}_{a n}(H)$ can be easily computed by numerically approximating (49) with sufficiently small $\mathcal{T}$ - recall that $\omega=\frac{1}{\mathcal{T}}$ quantifies the frequency at which moments attempt to switch. The Gibbs energy $G$ at the field value $H_{0}=2000 \mathrm{~A} / \mathrm{m}$, unnormalized density $\mu(G)=$ $e^{-G V / k T}$, and resulting local anhysteretic magnetization obtained with $\mathcal{T}=1.0 \times 10^{-13}$ sec, and relative thermal energies $k T / V=5.0 \times 10^{5}$ and $k T / V=7.14 \times 10^{6}$ are plotted in Figure 7 .

It is observed that when $k T / V$ is significant compared with $G$, thermal fluctuations produce switching between wells thus yielding a gradual anhysteretic transition between positive and negative saturation magnetizations. As $k T / V$ becomes increasingly small, the local anhysteretic magnetization $\bar{M}_{\text {an }}$ provided by (49) converges to

$$
\begin{aligned}
& \bar{M}(H)=\frac{H}{\eta}+M_{R} \delta \\
& \delta=\operatorname{sign}(H) .
\end{aligned}
$$

The limiting relation (60) can be interpreted as the locus of magnetization values which would occur in the absence of inclusions - which is manifested by $H_{c}=0$ in the local model.

Remark 5. We note that the local magnetization relation (29) obtained from the necessary condition $\frac{\partial G}{\partial M}=0$, with the statistical mechanics model for $G$, also yields an anhysteretic magnetization for

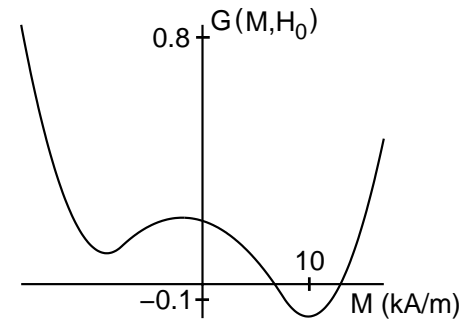

(a)

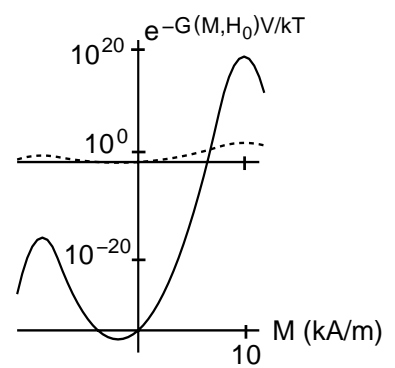

(b)

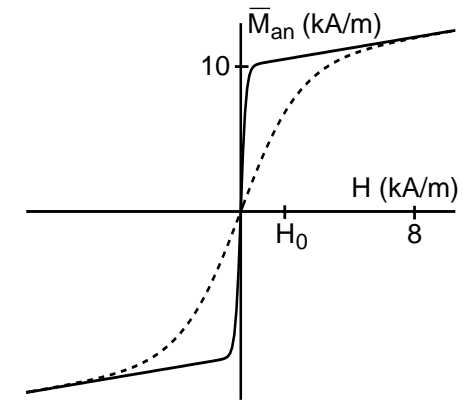

(c)

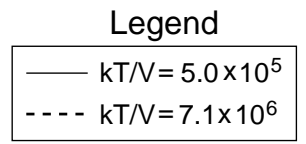

Figure 7: (a) Gibbs energy $G$, and (b) unnormalized Gaussian densities $\mu(G)=e^{-G V / k T}$ with $H_{0}=2000 \mathrm{~A} / \mathrm{m}$. (c) Anhysteretic magnetization $\bar{M}_{\text {an }}$ given by (49) with $\mathcal{T}=1.0 \times 10^{-13}$ sec. 
certain values of $\alpha$ and $a$. This is analogous to the Jiles-Atherton framework in which the Langevin expression (1) is employed to quantify $M_{a n}$. Due to its simplicity, however, we generally employ (60) as a kernel when characterizing the anhysteretic magnetization for regimes in which thermal relaxation is negligible.

\section{Macroscopic Magnetization Models}

The local magnetization models $(29,(32)$ or (49) were derived by constructing appropriate energy relations at the lattice level. For homogeneous materials with uniform effective fields, these relations hold throughout the material and hence will also provide macroscopic models. By construction, they exhibit the steep transitions depicted in Figures 4, 5 and 6 since moments are assumed to switch instantaneously once they achieve the energy required to overcome energy barriers. The models in this form prove adequate for characterizing the hysteretic behavior of certain materials which exhibit small demagnetizing factors, certain uniaxial wires and films or annealed toroidal specimens. Hysteresis loops exhibiting nearly instantaneous transitions due to these factors are illustrated for a uniaxial nickel-iron film, a magnetically annealed core of cobalt ferrous ferrite and manganesemagnesium ferrite on page 298 of Craig and Tebble [59]. However, the transitions provided by these local models are too steep to provide accurate characterization of general polycrystalline magnetic materials. To extend the local models, we consider certain parameters in the models to be statistically distributed to reflect variations in the lattice structure, exchange energies and grain orientations. The resulting macroscopic magnetization models accurately characterize both major and biased minor loops in a wide range of ferromagnetic materials.

\subsection{Statistical Mechanics Model}

An implicit assumption made when deriving the Helmholtz energy relation (18) used to construct the local magnetization models (29) and (49) is that the exchange energy $\Phi_{0}$ is constant throughout the lattice. This implies that the bias field $H_{h}=\frac{N \Phi_{0}}{2 V M_{s}}$ and Curie temperature $T_{c}=\frac{\Phi_{0}}{2 k}$ are constant which

yields a constant mean field coefficient $\alpha=\frac{H_{h}}{M_{s}}$ and constant coefficient $a(T)=\frac{H_{h} T}{T_{c}}$ in the models (29) and (49). However, for nonhomogeneous, polycrystalline materials with variable magnetization, this assumption is overly simplistic. For such materials, it is more reasonable to assume instead that $\Phi_{0}$ is statistically distributed which motivates the consideration of statistically distributed parameters in the macroscopic magnetization models. Additionally, material nonhomogeneities, variable grain orientations, nonuniform stress distributions, and variations due to texture motivate consideration of statistically distributed model parameters.

Because $\Phi_{0}$ quantifies the energy required to reorient a moment when the lattice is completely ordered, the assumption that $\Phi_{0}$ is statistically distributed implies that the exchange energy between spins or moments is distributed. Through (12), this implies that the exchange integral $\mathcal{J}$ is variable rather than constant as assumed for homogeneous materials. At the quantum level, the variability of $\mathcal{J}_{i j}$ employed in (5) is incorporated by modeling the overlap of electron wave functions whereas at the macroscopic level, it is incorporated by considering $H_{h}$ and $\alpha$ to be statistically distributed. We consider the construction of macroscopic mean field models which accommodate the microscopic variations in the exchange integral and lattice energy $\Phi_{0}$.

We first make the assumption that $\Phi_{0}$ is normally distributed about a mean value of $\bar{\Phi}_{0}$. If $N, V$ and $M_{s}$ remain constant, the bias field $H_{h}=\frac{N \Phi_{0}}{2 V M_{s}}$ will then be normally distributed with mean $\bar{H}_{h}$. 
A resulting macroscopic magnetization model is

$$
[M(H)](t)=C \int_{-\infty}^{\infty}\left[\bar{M}\left(H ; H_{h}, \xi\right)\right](t) e^{-\left(H_{h}-\bar{H}_{h}\right)^{2} / 2 b^{2}} d H_{h}
$$

where $b$ and $C$ are constants and $\bar{M}$ is specified by (29) or (49). Because $\alpha=\frac{H_{h}}{M_{s}}$, this is equivalent to employing effective fields

$$
H_{e}=H+\alpha M
$$

where $\alpha$ is normally distributed. This should be compared with a number of current hysteresis models which employ effective fields of the form (62) with fixed $\alpha$ (e.g., $[4,11,22]$ ).

More general effective fields can be incorporated if it is assumed that in addition to variations in $H_{h}$, the effective field $H_{e}$ at the domain level is normally distributed about the applied field $H$. Since $H_{e}=H+H_{I}$, where $H_{I}$ denotes the interaction field, this yields the macroscopic model

$$
[M(H)](t)=C \int_{-\infty}^{\infty} \int_{-\infty}^{\infty}\left[\bar{M}\left(H+H_{I} ; H_{h}, \xi\right)\right](t) e^{-H_{I}^{2} / 2 b^{2}} e^{-\left(H_{h}-\bar{H}_{h}\right)^{2} / 2 b^{2}} d H_{I} d H_{h}
$$

where $b^{2}$ determines the variance about $H$.

\subsection{Piecewise Quadratic Helmholtz Relations}

For the model derived using the piecewise quadratic Helmholtz energy (25), we incorporate lattice variations due to nonhomogeneities, polycrystallinity and material inclusions by considering the local coercive field $H_{c}$ to be stochastically distributed. To enforce $H_{c} \geq 0$, we make the initial assumption that $H_{c}$ satisfies a lognormal distribution with density

$$
\nu_{1}\left(H_{c}\right)=c_{1} \exp \left\{-\left[\frac{\ln \left(H_{c} / \bar{H}_{c}\right)}{2 c}\right]^{2}\right\}
$$

where $c, c_{1}$ and $\bar{H}_{c}$ are positive constants. It is illustrated in [9], where this distribution is considered in the context of Preisach models, that if $c$ is small compared with $\bar{H}_{c}$, the mean and variance of $f$ have the approximate values

$$
\left\langle H_{c}\right\rangle \approx \bar{H}_{c} \quad, \quad \sigma \approx 2 \bar{H}_{c} c .
$$

The macroscopic magnetization model based on this distribution of $H_{c}$ is

$$
[M(H)](t)=\int_{0}^{\infty}\left[\bar{M}\left(H ; H_{c}, \xi\right)\right](t) \nu_{1}\left(H_{c}\right) d H_{c}
$$

where $\bar{M}$ is given by (32) or (49). The relations (65) can be used to obtain initial parameter estimates from attributes of measured data. For certain materials, the distribution of $H_{c}$ can be taken as Gaussian; however, positivity should still be enforced in the integration limits.

To incorporate variability in the exchange energy $\Phi_{0}$, we consider the effective field $H_{e}$ to be normally distributed about the applied field $H$. When combined with (66), this yields the macroscopic magnetization model

$$
[M(H)](t)=C \int_{0}^{\infty} \int_{-\infty}^{\infty}\left[\bar{M}\left(H+H_{I} ; H_{c}, \xi\right)\right](t) e^{-H_{I}^{2} / 2 b^{2}} e^{-\left[\ln \left(H_{c} / \bar{H}_{c}\right) / 2 c\right]^{2}} d H_{I} d H_{c}
$$

where $C$ and $b$ are positive constants and $\bar{M}$ is given by (32) or (49). When the kernel $\bar{M}$ is computed using (49), the model incorporates certain relaxation mechanisms including magnetic after-effects. However, it does not incorporate elastic effects or eddy current dynamics in this formulation so it should be employed in low frequency regimes. 


\subsection{General Density Formulation}

The macroscopic model formulations (63) and (67) are based on a priori assumptions regarding the normal or lognormal nature of underlying densities. In certain cases, the normal behavior of parameters can be argued using statistical theory based on the central limit theorem; e.g., see [9]. In general, however, the choice of normal or lognormal distributions is based solely on mathematical attributes rather than physical or energy principles. These mathematical assumptions can be avoided by formulating the macroscopic models in terms of general densities to be estimated through a least squares fit to data.

To illustrate, let $\nu_{1}$ and $\nu_{2}$ designate the densities respectively associated with local coercive and effective fields. To satisfy physical criteria, we assume that $\nu_{1}$ and $\nu_{2}$ satisfy the conditions

$$
\begin{array}{ll}
\text { (i) } & \nu_{1}(x) \text { defined for } x>0 \\
\text { (ii) } & \nu_{2}(-x)=\nu_{2}(x) \\
\text { (iii) } & \left|\nu_{1}(x)\right| \leq c_{1} e^{-a_{1} x},\left|\nu_{2}(x)\right| \leq c_{2} e^{-a_{2} x}
\end{array}
$$

for positive $a_{1}, a_{2}, c_{1}, c_{2}$. The restricted domain in (i) reflects the fact that the coercive field $H_{c}$ is positive whereas the symmetry enforced in the effective field through (ii) yields the symmetry observed in low-field Rayleigh loops. Hypothesis (iii) incorporates the physical observation that the coercive and interaction fields decay as a function of distance and guarantees that integration against the piecewise linear kernel yields finite magnetization values.

For the piecewise quadratic Helmholtz energy (25), The resulting macroscopic magnetization model is then given by

$$
\begin{aligned}
{[M(H)](t) } & =\int_{0}^{\infty} \int_{-\infty}^{\infty} \nu_{1}\left(H_{c}\right) \nu_{2}\left(H_{I}\right)\left[\bar{M}\left(H+H_{I} ; H_{c}, \xi\right)\right](t) d H_{I} d H_{c} \\
& =\int_{0}^{\infty} \int_{-\infty}^{\infty} \nu\left(H_{c}, H_{I}\right)\left[\bar{M}\left(H+H_{I} ; H_{c}, \xi\right)\right](t) d H_{I} d H_{c}
\end{aligned}
$$

where $\bar{M}$ is specified by (31) or (49). Formulation in terms of the product density $\nu$ is more general whereas retention of the components $\nu_{1}$ and $\nu_{2}$ can facilitate subsequent implementation.

Remark 6. A comparison of (69) with (2) indicates the manner through which the framework provides an energy basis for certain extended Preisach models as detailed in [32,33]. Techniques analogous to those developed for Preisach models [27] have been developed to identify the general density $\nu$ in an efficient manner [60,61], thus exploiting the similarity between the two frameworks. As detailed in Sections 1 and 5, the formulation (69) is advantageous over classical Preisach models in the manner through which it relaxes reversibility, deletion, and congruency criteria and incorporates temperature and rate-dependencies in the basis $\bar{M}$ rather than in the parameters $\nu$. For these reasons, it provides an energy basis for certain extended Preisach models [9].

\subsection{Anhysteretic Magnetization Model}

It was illustrated in Section 3.2 that for the piecewise quadratic Gibbs energy, the local anhysteretic magnetization $\bar{M}_{a n}$ followed naturally from (49) for thermally active regimes or (60) in the absence of thermal after-effects or relaxation. The global anhysteretic model follows directly from the general hysteresis model (67) or (69) but can be simplified substantially since coercive fields play no role in the anhysteretic material behavior. 
We consider first the anhysteretic model for the a priori density choices

$$
\begin{aligned}
& \nu_{1}\left(H_{c}\right)=\frac{\widetilde{c}_{1}}{I_{1}} e^{-\left[\ln \left(H_{c} / \bar{H}_{c}\right) / 2 c\right]^{2}} \\
& \nu_{2}\left(H_{I}\right)=\frac{\widetilde{c}_{2}}{b \sqrt{2 \pi}} e^{-H_{I}^{2} / 2 b^{2}}
\end{aligned}
$$

where $c_{1}=\widetilde{c}_{1} / I_{1}$ and $c_{2}=\widetilde{c}_{2} / b \sqrt{2 \pi}$ are expressed in terms of the normalization constants

$$
I_{1}=\int_{0}^{\infty} e^{-\left[\ln \left(H_{c} / \bar{H}_{c}\right) / 2 c\right]^{2}} d H_{c} \quad, \quad b \sqrt{2 \pi}=\int_{-\infty}^{\infty} e^{-H_{I}^{2} / 2 b^{2}} d H_{I}
$$

The anhysteretic magnetization is then given by

$$
\begin{aligned}
M_{a n}(H) & =\frac{\widetilde{c}_{1}}{I_{1}} \cdot \frac{\widetilde{c}_{2}}{b \sqrt{2 \pi}} \int_{0}^{\infty} \int_{-\infty}^{\infty} \bar{M}\left(H+H_{I}\right) e^{-\left[\ln \left(H_{c} / \bar{H}_{c}\right) / 2 c\right]^{2}} e^{-H_{I}^{2} / 2 b^{2}} d H_{I} d H_{c} \\
& =\widetilde{C} \int_{-\infty}^{\infty} \bar{M}\left(H+H_{I}\right) e^{-H_{I}^{2} / 2 b^{2}} d H_{I}
\end{aligned}
$$

where

$$
\widetilde{C}=\frac{\widetilde{c}_{1} \widetilde{c}_{2}}{b \sqrt{2 \pi}} .
$$

If solely quantifying anhysteretic material behavior, one can treat the constant $\widetilde{C}$ as a material parameter to be identified whereas if correlating modeled hysteretic and anhysteretic properties, one should identify the constants $\widetilde{c}_{1}$ and $\widetilde{c}_{2}$. In the absence of thermal after-effects, the local relation (60) yields

$$
\begin{aligned}
& \bar{M}\left(H+H_{I}\right)=\frac{H+H_{I}}{\eta}+M_{R} \delta \\
& \delta=\operatorname{sign}\left(H+H_{I}\right)
\end{aligned}
$$

whereas the kernel (49) can be employed if thermal activation is significant.

Additional generality can be obtained through the formulation

$$
M_{a n}(H)=\int_{-\infty}^{\infty} \bar{M}\left(H+H_{I}\right) \nu_{2}\left(H_{I}\right) d H_{I}
$$

where $\nu_{2}$ is a general density satisfying the assumption (68). As with the parameterized formulation, normalization constants must be accounted for if comparing the anhysteretic model (75) and hysteresis model (69) - this reflects the price paid for employing unnormalized density formulations to simplify notation.

\subsection{Model Implementation}

Two issues must be addressed when implementing the hysteresis models (63) or (67); (i) approximation of the integrals, and (ii) efficient implementation of the conditional relations (32). Because both issues are crucial for providing algorithms that permit efficient system design and real-time control implementation, we summarize pertinent details. For simplicity, we focus on the implementation of (67) and note that analogous constructs exist for (63). 


\subsubsection{Quadrature Techniques}

The integrals can be approximated either on the original infinite and semi-infinite domains or on finite domains determined by the exponential decay properties of the integrands. On the infinite domain, Gauss-Hermite quadrature formulae apply whereas Gauss-Laguerre points and weights apply for the semi-infinite integrals [62]. As illustrated in Figures 8(a) and 8(c), the exponential decay of the densities can also be employed to determine finite intervals where Gauss-Legendre formulae are accurate.

In all cases, approximation of (67) yields

$$
[M(H)](t)=C \sum_{i=1}^{N_{i}} \sum_{j=1}^{N_{j}}\left[\bar{M}\left(H_{I_{j}}+H ; H_{c_{i}}, \xi_{j}\right)\right](t) e^{-H_{I_{j}}^{2} / 2 b^{2}} e^{-\left[\ln \left(H_{c_{i}} / \bar{H}_{c}\right) / 2 c\right]^{2}} v_{i} w_{j}
$$

where $H_{I_{j}}, H_{c_{i}}$ denote the abscissas and $v_{i}, w_{j}$ are weights associated with the respective quadrature formulae. At $H=0, M=0$, the initial moment distribution $\xi_{j}$ corresponds with the quadrature points as illustrated in Figure 8(b).

To further illustrate, we consider the construction of Gauss-Legendre points and weights on the interval $[-L, L]$ using a 4 point composite quadrature rule. On each subinterval $\left[h_{j-1}, h_{j}\right]$, where $h_{j}=-L+j h$, the quadrature points and weights are

$$
\begin{array}{lll}
H_{I_{q 1}}=h_{q-1}+h\left[\frac{1}{2}-\frac{\sqrt{15+2 \sqrt{30}}}{2 \sqrt{35}}\right] & , & w_{q 1}=\frac{49 h}{12(18+\sqrt{30})} \\
H_{I_{q 2}}=h_{q-1}+h\left[\frac{1}{2}-\frac{\sqrt{15-2 \sqrt{30}}}{2 \sqrt{35}}\right] & , & w_{q 2}=\frac{49 h}{12(18-\sqrt{30})} \\
H_{I_{q 3}}=h_{q-1}+h\left[\frac{1}{2}+\frac{\sqrt{15-2 \sqrt{30}}}{2 \sqrt{35}}\right] & , & w_{q 3}=\frac{49 h}{12(18-\sqrt{30})} \\
H_{I_{q 4}}=h_{q-1}+h\left[\frac{1}{2}+\frac{\sqrt{15+2 \sqrt{30}}}{2 \sqrt{35}}\right] & & w_{q 4}=\frac{49 h}{12(18+\sqrt{30})}
\end{array}
$$

For $N_{q}=2$ intervals, and hence $N_{j}=8$, the quadrature points specified by (77) are depicted in Figure 8(b).

The use of a similar relation to approximate the coercive field integral yields the double sum (76) which must be evaluated when computing a magnetization value for each input field value. From (32), it is observed that for each field value $H_{I_{j}}$, it is necessary to determine whether a transition has occurred relative to the coercive value $H_{c_{i}}$. This yields $N_{i} \times N_{j}$ conditions to be checked for each input value. While this can be easily accomplished using an if-then construct, implementation in this manner diminishes significantly the efficiency of the algorithm. This motivates consideration of an algebraic technique for evaluating the conditional statements.

\subsubsection{Implementation Algorithm - Hysteresis Model with Negligible After-Effects}

To retain the history of whether or not effective field values $H_{I_{j}}=H+H_{I_{j}}$ have switched due to encounters with coercive field values $H_{c_{i}}$, we employ (31) to motivate the matrix formulation

$$
\bar{M}=\frac{H}{\eta}+M_{R} \Delta\left(H ; H_{c}, H_{I}\right)
$$




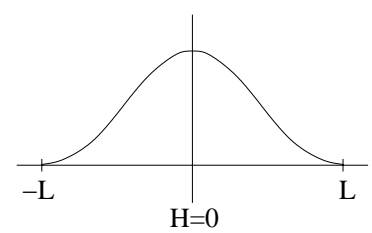

(a)

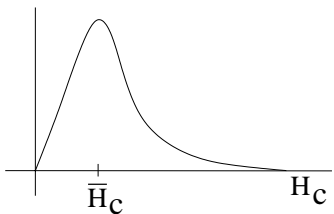

(c)

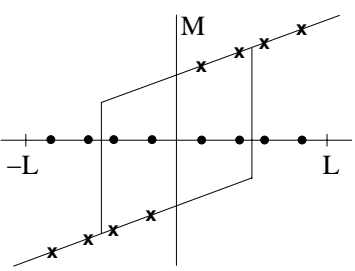

(b)

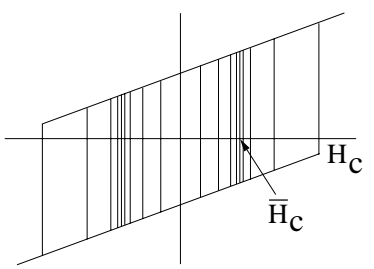

(d)

Figure 8: (a) Decay exhibited by the effective field $H_{I}$ having the density $e^{-H_{I}^{2} / 2 b^{2}}$ and truncated domain $[-L, L]$. (b) Gaussian quadrature points $\bullet$ and initial local magnetization values $\xi_{j}$ for $N_{j}=8$. (c) Lognormal density $\nu_{1}\left(H_{c}\right)=c_{1} e^{-\left[\ln \left(H_{c} / \bar{H}_{c}\right) / 2 c\right]^{2}}$ given by (64). (d) Distribution of hysteresis kernels having coercive fields $H_{c}$.

where $\Delta=1$ if evaluating on the upper branch of the hysteron and $\Delta=-1$ if on the lower branch. For the evaluation of $(77), \Delta$ is an $N_{i} \times N_{j}$ matrix whose $i j$ th component specifies whether $H_{I_{j}}$ has reached the coercive value $H_{c_{i}}$. We also define the following matrices

$$
\begin{gathered}
\Delta_{\text {init }}=\left[\begin{array}{rrrrrr}
-1 & \cdots & -1 & 1 & \cdots & 1 \\
\vdots & & \vdots & \vdots & & \vdots \\
-1 & \cdots & -1 & 1 & \cdots & 1
\end{array}\right]_{N_{i} \times N_{j}}, \mathcal{H}_{c}=\left[\begin{array}{lll}
H_{c_{1}} & \cdots & H_{c_{1}} \\
\vdots & & \vdots \\
H_{c_{N_{i}}} & \cdots & H_{c_{N_{i}}}
\end{array}\right]_{N_{i} \times N_{j}} \\
\mathcal{H}_{k}=\left[\begin{array}{lll}
H_{k}+H_{I_{1}} & \cdots & H_{k}+H_{I_{N_{j}}} \\
\vdots & & \vdots \\
H_{k}+H_{I_{1}} & \cdots & H_{k}+H_{I_{N_{j}}}
\end{array}\right]_{N_{i} \times N_{j}}
\end{gathered}
$$

and weight vectors

$$
\begin{aligned}
W^{T} & =\left[w_{1} e^{-H_{I_{1}}^{2} / 2 b^{2}}, \cdots, w_{N_{j}} e^{-H_{I_{N_{j}}}^{2} / 2 b^{2}}\right]_{1 \times N_{j}} \\
V^{T} & =\left[v_{1} e^{-\left[\ln \left(H_{c_{1}} / \bar{H}_{c}\right) / 2 c\right]^{2}}, \cdots, v_{N_{i}} e^{-\left[\ln \left(H_{c_{N_{i}}} / \bar{H}_{c}\right) / 2 c\right]^{2}}\right]_{1 \times N_{i}} .
\end{aligned}
$$

Here $H_{k}=H\left(t_{k}\right)$ is the $k$ th value of the input field. The magnetization $M_{k} \approx M\left(H_{k}\right)$ is specified by Algorithm 1. Here sgn denotes the signum function, .* indicates componentwise multiplication, and

$$
C=c_{1} \cdot c_{2}=\frac{\widetilde{c}_{1}}{I_{1}} \cdot \frac{\widetilde{c}_{2}}{b \sqrt{2 \pi}}
$$

follows from (71). The first step in the for-loop updates $\Delta$ by incorporating the status of previous coercive field switches. We note that by employing algebraic matrix operations to evaluate and 
incorporate $\Delta$, the efficiency of the algorithm is improved by a factor of more that 100 over algorithms utilizing conditional evaluations. This efficiency is crucial for system design and real-time control implementation.

\section{Algorithm 1.}

$$
\begin{aligned}
& \begin{array}{l}
\Delta_{\text {prev }}=\Delta_{\text {init }} \\
\text { for } k=1: N_{k} \\
\qquad=\operatorname{sgn}\left(\mathcal{H}_{k}+\mathcal{H}_{c} * \Delta_{\text {prev }}\right) \\
\bar{M}=\frac{1}{\eta} \mathcal{H}_{k}+M_{R} \Delta \\
M_{k}=C V^{t} \bar{M} W \\
\Delta_{\text {prev }}=\Delta
\end{array} \\
& \text { end }
\end{aligned}
$$

\subsubsection{Implementation Algorithm - Anhysteretic Model}

The implementation of the discretized anhysteretic model is significantly easier than implementation of the hysteresis model since it does not require updating of $\Delta$ to retain a history of moment switches due to local coercive fields. One can employ either the matrices and vectors defined in (79) and (80) for the hysteresis model, or a reduced set of vectors which reflects the fact that the coercive density integrates to the constant $I_{1}$ defined in (71). The two equivalent approaches are illustrated in Algorithms 2 and 3 where $\widetilde{C}=I_{1} C$ and

$$
h_{k}=\left[H_{k}+H_{I_{1}}, \cdots, H_{k}+H_{I_{N_{j}}}\right]_{1 \times N_{j}}
$$

in Algorithm 3. Algorithm 2 retains the direct correlation with the hysteresis model whereas Algorithm 3 is more efficient to implement since it requires vector rather than matrix multiplication.

\section{Algorithm 2.}

$$
\begin{gathered}
\text { for } k=1: N_{k} \\
\Delta=\operatorname{sgn}\left(\mathcal{H}_{k}\right) \\
\bar{M}_{a n}=\frac{1}{\eta} \mathcal{H}_{k}+M_{R} \Delta \\
M_{k}=C V^{T} \bar{M}_{a n} W
\end{gathered}
$$

end

\section{Algorithm 3.}

$$
\text { for } \begin{aligned}
k=1: N_{k} \\
\Delta=\operatorname{sgn}\left(h_{k}\right) \\
\bar{M}_{a n}=\frac{1}{\eta} h_{k}+M_{R} \Delta \\
M_{a n_{k}}=\widetilde{C} \bar{M}_{a n} W
\end{aligned}
$$

end

\section{Model Properties and Validation}

To illustrate attributes of the model, we consider the characterization of steel under zero prestress conditions using data from [10]. This demonstrates a variety of regimes including quantification of the anhysteretic magnetization and the prediction of symmetric minor loop behavior using the model with parameters obtained from the symmetric major loop. To further illustrate the manner 
through which the framework characterizes anhysteretic material behavior, we numerically simulate the experimental process in which a decaying AC field is applied at a DC offset to demonstrate that the resulting magnetization coincides with that predicted by the anhysteretic model. In the third example, we illustrate the response of the model to a step input to demonstrate its capability for quantifying the effects of thermal activation. Finally, we note that additional examples illustrating the capability of the model to accurately characterize biased minor loop behavior can be found in $[63,64]$ and the characterization of strains in a Terfenol-D transducer is illustrated in [36].

\subsection{Experimental Validation for Steel: Anhysteretic and Biased Minor Loop Behavior}

We consider data from a steel specimen having a length of $6 \mathrm{~cm}$ and cross-sectional area of $1 \mathrm{~cm}$ as reported by Jiles and Atherton [10]. The composition (\% by weight) of the sample was C (0.08), Mn (1.98), S (0.08), P (0.015), Cu (0.055) and Mo (0.235). We consider hysteretic and anhysteretic data collected under $\sigma=0$ prestress conditions which is plotted in Figures 9(a) and 9(b).

\section{Anhysteretic Model}

The anhysteretic model (72) is more fundamental than the full hysteresis model (67), since it does not incorporate local coercive fields, so we consider it first. The parameters $M_{R}, \widetilde{C}, \eta$ and $b$ have the following physical interpretations. The local remanence value $M_{R}$ and constant $\widetilde{C}$ both scale the height of the curve and are constructed to yield correct saturation values. The parameter $\eta$ asymptotically represents the reciprocal slope $\frac{d H}{d M}$ at field reversal and an initial value can be obtained from the slope of the data at $H_{\max }$. The variance $b$ quantifies the degree of pre-remanence switching in the hysteresis model which corresponds to the curvature of the anhysteretic model in low drive regimes.

A least squares fit to the data yielded the values for $M_{R}, \widetilde{C}, \eta$ and $b$ summarized in Table 1 . A comparison between the resulting model fit and data in Figure 9(a) illustrates that the anhysteretic model (72) accurately characterizes the material behavior through the drive range.

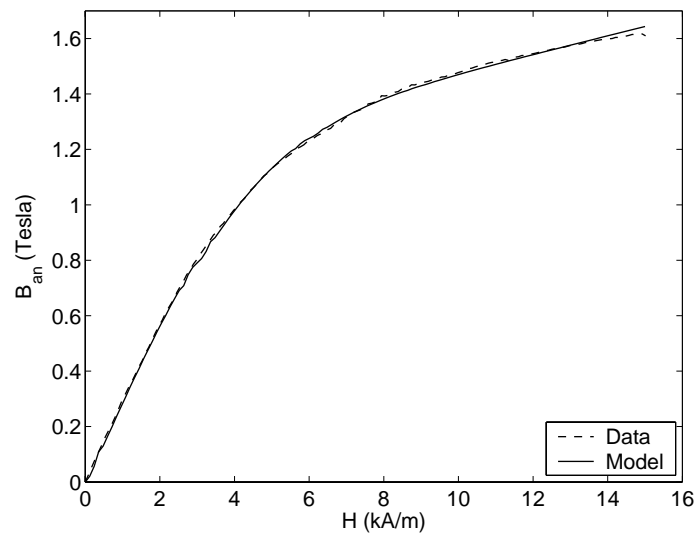

(a)

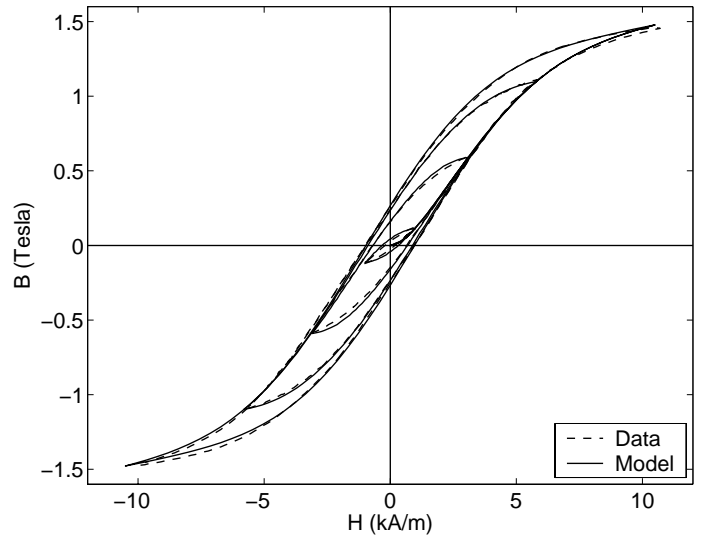

(b)

Figure 9: Steel data from [10] collected under $\sigma=0$ prestress conditions. (a) Anhysteretic data and model fit provided by (72). (b) Hysteresis data, major loop model fit provided by (67), and minor loop predictions. 


\begin{tabular}{l|cccccc}
\hline \hline Parameters & $\eta$ & $M_{R}(\mathrm{~A} / \mathrm{m})$ & $b(\mathrm{~A} / \mathrm{m})$ & $\widetilde{C}$ & $\bar{H}_{c}(\mathrm{~A} / \mathrm{m})$ & $c(\mathrm{~A} / \mathrm{m})$ \\
\hline Values & 6.5 & $5.4 \times 10^{3}$ & 3521.4 & 0.0190 & 250 & 0.75 \\
\hline
\end{tabular}

Table 1: Parameter values identified for the anhysteretic model (72) and hysteresis model (67).

\section{Hysteresis Model}

The full hysteresis model additionally requires the estimation of the mean coercive field $\bar{H}_{c}$ and variance $c$. Whereas an initial estimate for the former can be obtained directly from the coercivity of the data, the parameter $c$ which quantifies the variability at coercivity due to material nonhomogeneities, is usually prescribed a qualitative rather than quantitative interpretation. For example, materials exhibiting a steep transition at coercivity will have small variances when compared with materials such as the steel data plotted in Figure 9(b) which exhibits a gradual transition at $H=H_{c}$.

To complete the model, the measured coercive field $H_{c}=910 \mathrm{~A} / \mathrm{m}$ was used as an initial value and the values of $\bar{H}_{c}$ and $c$ compiled in Table 1 were estimated through a least squares fit to the symmetric major loop data. Measured periodic fields having lower amplitudes were subsequently input to the model - using the same parameter values - to obtain the symmetric minor loop predictions which are also plotted in Figure 9(b). It is observed that the model accurately characterizes the hysteretic material behavior throughout the drive regime, including the approximately quadratic Rayleigh loop behavior at low input fields.

\subsection{Numerical Simulation of Anhysteretic Behavior}

To further illustrate the manner through which the anhysteretic magnetization is quantified by this framework, we numerically simulate the experimental procedure used to obtain $M_{a n}$ using the full hysteresis model (67), and compare with the value predicted by the anhysteretic model (72). Specifically, we applied the periodic and subsequently decaying AC field depicted in Figure 10(a) to the model (67) to simulate the experimental procedure used to obtain $M_{a n}$ or $B_{a n}$ at the DC field $H_{0}=2000 \mathrm{~A} / \mathrm{m}$. The parameter values from Table 1 were employed so the result, $B_{a n}=0.5544$ Tesla, which is plotted as $*$ in Figure 10(b), is representative of steel. A comparison with the

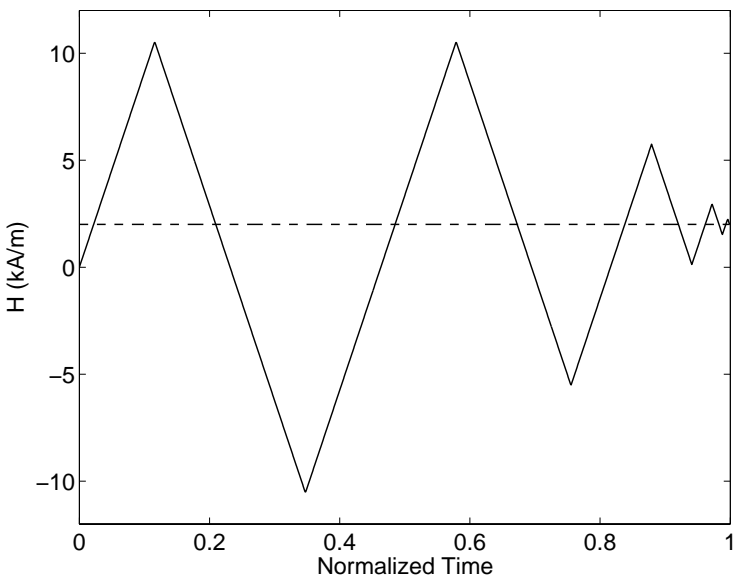

(a)

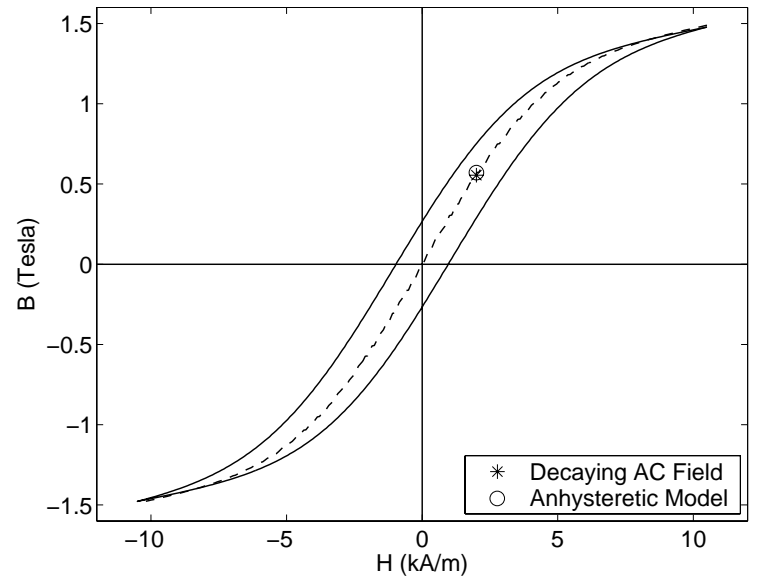

(b)

Figure 10: (a) Field input to the hysteresis model (67) to generate the hysteretic response and anhysteretic value at $H_{0}=2000 \mathrm{~A} / \mathrm{m}$. (b) Value of $B_{\text {an }}$ generated by the decaying $\mathrm{AC}$ field $(*)$ and anhysteretic model (o), and full anhysteretic curve (-- ) given by (72). 
corresponding value of $B_{a n}=0.5704$ Tesla predicted by the anhysteretic model (72), which is denoted by $\circ$, illustrates that the two approaches yield identical results to within reasonable precision. The locus of points computed using (72) completes the comparison between the predicted anhysteretic and hysteretic responses for the material. Hence the modeling framework developed to quantify hysteresis in ferromagnetic materials also quantifies the anhysteretic response in a natural manner.

\subsection{Experimental Validation for Nickel: Biased Minor and Reversible Post- Switching Behavior}

To illustrate the capability of the model to characterize biased mior loop behavior, we consider data collected from a rod comprised of Nickel 200. As detailed in [65], the rod had a diameter of $0.0635 \mathrm{~cm}$ $(1 / 4 \mathrm{in})$, length of $6.858 \mathrm{~cm}$ ( $2.7 \mathrm{in})$, and was employed in a water-cooled transducer analogous to the Terfenol-D design depicted in Figure 2. Data was collected at a rate of $10 \mathrm{mHz}$ to minimize eddy current losses.

The kernel (49), which incorporates thermal relaxation, was employed in the magnetization model and general densities $\nu_{1}$ and $\nu_{2}$ were identified using the least squares techniques detailed in [61] for the analogous ferroelectric model. When implementing the model in optimization routines, the measured experimental field $H$, plotted in Figure 11(a), was employed as input to the model to maintain experimental rates and time scales. The resulting magnetization data and model fit are shown in Figure 11(b)-(e). The time history in Figure 11(b) illustrates the fact that because the nested minor loop data comprises a large percentage of the total data set, the optimization routine determines density values which yield greater accuracy in the minor loops than in the post-switching region of the major loop. The slight nonclosure of modeled minor loops results from the relaxation values prescribed during the optimization process to accommodate similar behavior in the measured data.

For fields higher in magnitude than approximately $6 \mathrm{kA} / \mathrm{m}$, the model incorporates the reversible post-switching behavior of the material due to the form of the energy-based kernel. The characterization of these effects using a Preisach approach requires extensions of the type detailed in [9] since classical Preisach hysterons have zero slope. Similarly, an extended formulations of the Jiles-Atherton model is required to incorporate this effect.

The accuracy exhibited in Figure 11(b) is important for model-based control design since characterization and compensation in this context are typically posed as a functions of time. Further details regarding the utility of the framework for model-based control design are provided in Section 6 .

\subsection{Numerical Simulation of Relaxation Behavior}

One manifestation of relaxation effects is the nonclosure of biased minor loops observed in Figure 12. To further illustrate the manner through which the model characterizes magnetic after-effects, the discontinuous step input shown in Figure 12(a) was input to the model. The resulting magnetization, plotted in Figure 12(b), exhibits both an associated discontinuity and creep or after-effects due to the modeled thermal activation mechanisms. Quantification of this phenomenon is important both for fundamental material modeling and characterization of transducers required to hold a precise set point over time scales comparable with relaxation times.

For the classes of macroscopic models discussed in Section 1, extended Preisach formulations incorporating Arrhenius behavior will characterize this behavior whereas the Jiles-Atherton, Globus, and Stoner-Wohlfarth formulations do not presently address this phenomenon. The inherent incorporation of thermal activation mechanisms constitutes an advantage of the homogenized energy framework. 


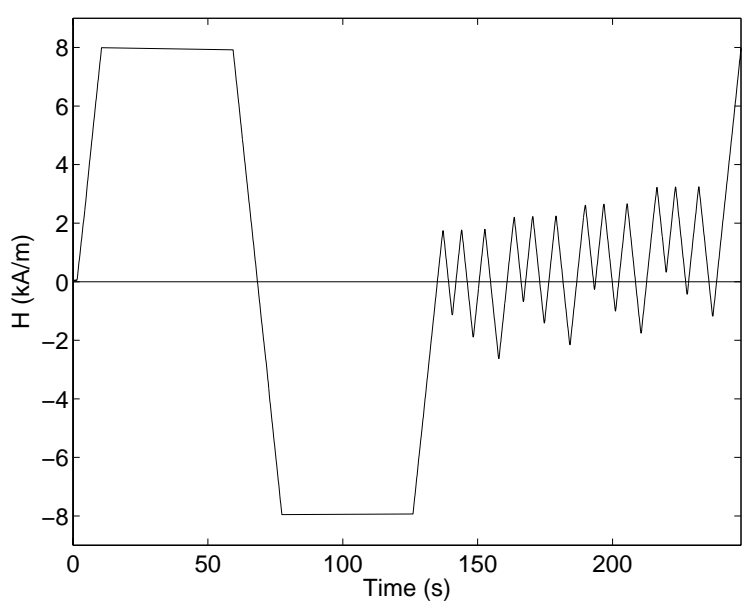

(a)

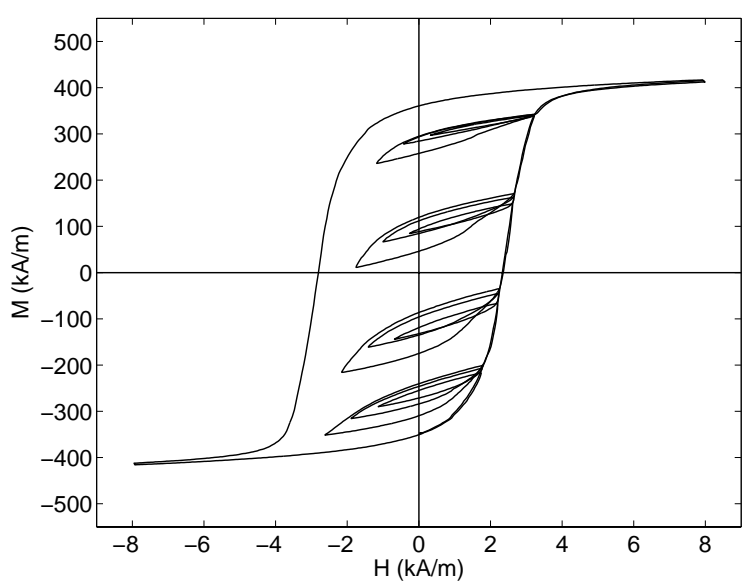

(c)

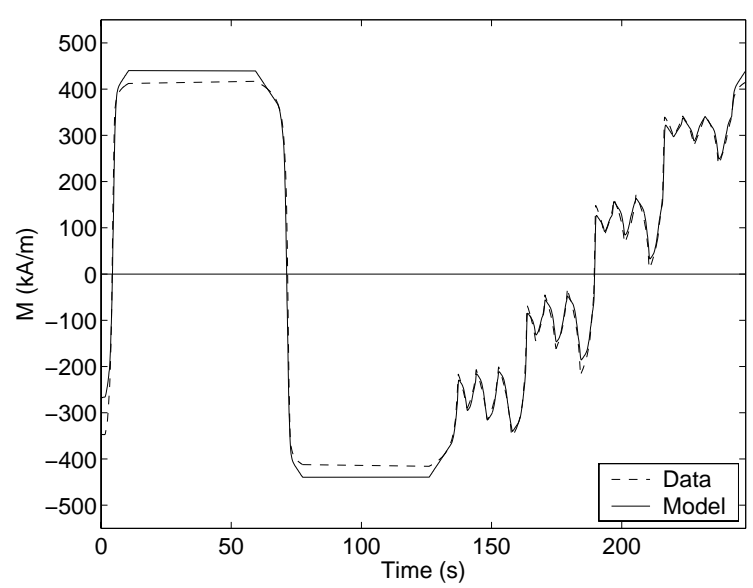

(b)

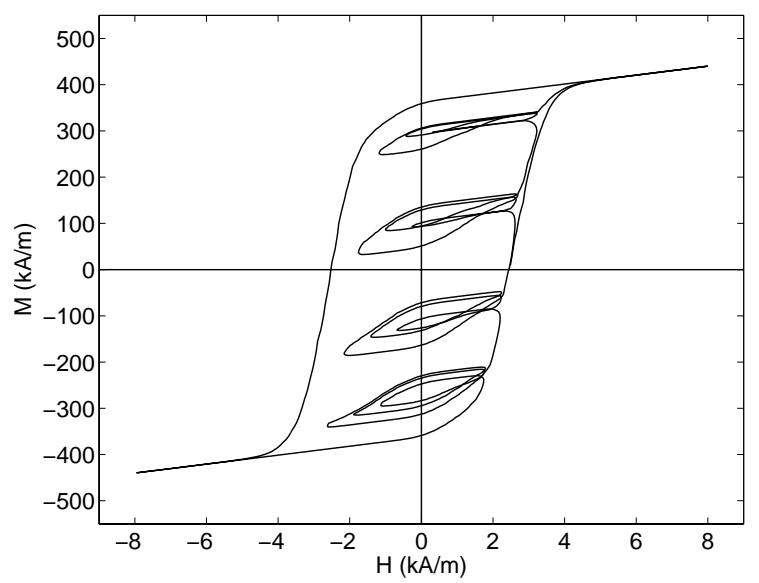

(d)

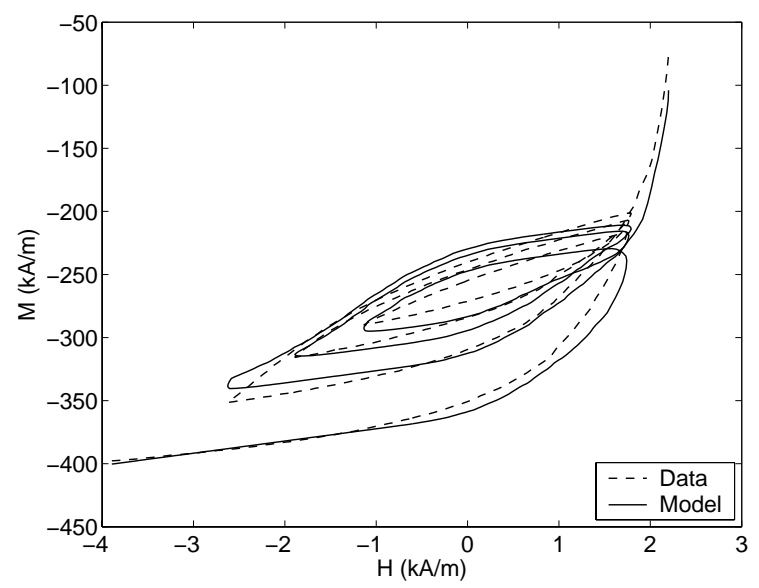

(e)

Figure 11: (a) Field input, (b) Nickel 200 data and model as a function of time, (c) Nickel 200 $H-M$ data, (d) $H-M$ model fit, and (e) comparison between data and model in the first minor loop following negative remanence. 


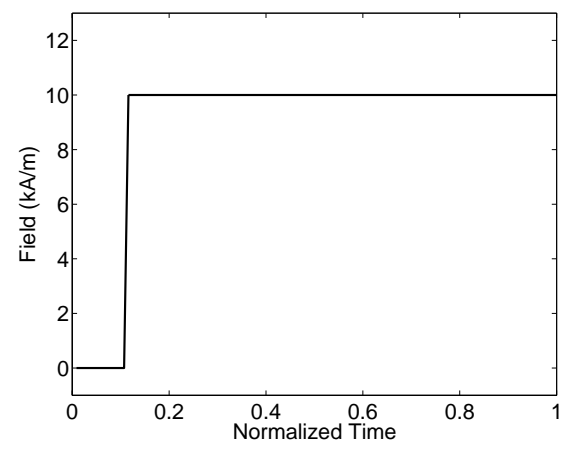

(a)

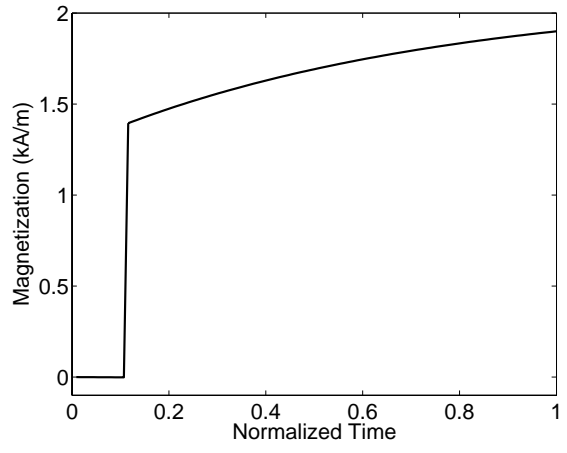

(b)

Figure 12: (a) Input field $H$ and (b) response of the model (69) employing the kernel (49) which incorporates thermal activation.

\section{Concluding Remarks}

The model presented here provides a framework for characterizing hysteretic and anhysteretic behavior of a broad range of ferromagnetic compounds. The approach combines energy analysis at the lattice level with stochastic homogenization techniques to construct macroscopic models which accurately characterize the nonlinear $H-M$ or $H-B$ material behavior while remaining sufficiently efficient to permit subsequent transducer design or model-based control designs having the potential for real-time implementation. At the lattice level, exchange and magnetostatic energy relations are employed in a mean field framework to construct a temperature-dependent Gibbs energy relation $G$ which quantifies the transition from ferromagnetic to paramagnetic behavior as temperatures are increased through the Curie temperature $T_{c}$. For fixed temperature regimes, Taylor expansion about equilibria yields a piecewise quadratic Gibbs relation which is highly efficient to implement. For regimes in which magnetic after-effects (thermal relaxation) are negligible, the local average magnetization $\bar{M}$ at the lattice level is determined from the necessary condition $\frac{\partial G}{\partial M}=0$ whereas Boltzmann principles are employed to balance $G$ with the relative thermal energy $k T / V$ for operating regimes in which relaxation times are significant compared with drive frequencies.

To construct macroscopic models utilizing the energy-based kernels or hysterons $\bar{M}$, we assume that bias fields $H_{h}$, local coercive fields $H_{c}$, and interaction fields $H_{I}$ are manifestations of underlying distributions rather than constant parameters or inputs. This yields constitutive relations which guarantee minor loop closure once accommodation and after-effects are completed, incorporate relaxation and certain temperature-dependencies, incorporate reversible effects, and yield noncongruency in certain operating regimes. The model is also highly efficient to implement thus facilitating future incorporation in design and control algorithms. Finally, the framework incorporates mechanisms common to a number of presently employed macroscopic models.

Jiles-Atherton: The Ising relation (29) obtained by minimizing the Gibbs energy constructed through statistical mechanics tenets is analogous, and agrees through first-order terms, with the Langevin relation (1) employed in the Jiles-Atherton theory to quantify the anhysteretic magnetization. The difference between the theories lies in the manner through which energy relations are constructed and losses are incorporated.

Globus and Stoner-Wohlfarth: The energy-based hysterons $\bar{M}$ provided by the proposed model are also analogous to those provided by the Globus and Stoner-Wohlfarth models. The present theory differs from the original Globus and Stoner-Wohlfarth theories due to its formulation in terms of the exchange energy which incorporates moment interactions. It also differs from the Stoner-Wohlfarth 
theory in that it does not incorporate anisotropy energies.

Preisach: The theory bears the greatest resemblance to extended Preisach theories and the formulation (69) in terms of general densities provides an energy basis for certain extended Preisach models. The primary differences between the proposed framework and classical Preisach theory are the following. (i) As detailed in Section 5.1, certain parameters such as the local average coercive field $\bar{H}_{c}$ and reciprocal slope $\eta$ at $H_{\max }$ can be directly correlated with properties of the data to facilitate model construction and updating. This is analogous to interpretations proposed in [9] for Preisach models with a priori density specifications. (ii) Temperature and relaxation mechanisms are incorporated in the basis or kernel $\bar{M}$ of (67) or (69) rather than the densities or weights as is typically the case for Preisach formulations. This eliminates the need for vector-valued measures or lookup tables for general operating conditions. (iii) As illustrated in Figure 5, the proposed framework employing the kernel (29) relaxes the criterion of congruency and hence is analogous to certain moving Preisach models [9]. (iv) The model also automatically incorporates reversible effects after switching and hence does not required the Preisach extensions detailed in [9] to incorporate reversibility.

We note that the framework developed here for ferromagnetic materials is based on theory quantifying phase transitions in shape memory alloys (SMA) [66-69] which has subsequently been extended to ferroelectric materials $[61,70]$. Hence the framework provides a unified methodology for modeling constitutive nonlinearities and hysteresis in ferroelectric, ferromagnetic and ferroelastic materials $[63,64]$. This directly facilitates the construction of unified characterization techniques for ferroic compounds and the development of unified model-based control designs for ferroic transducers. It also provides a framework which may facilitate the construction of models for hybrid transducers employing multiple components (e.g., Terfenol-D and PZT) or the characterization of newly developed and proposed materials such as ferromagnetic shape memory alloys (FSMA) which exhibit attributes common to multiple classes of the compounds.

As noted in Section 2, exchange, magnetostatic, magnetoelastic and anisotropy energy components contribute to the total energy in ferromagnetic materials. The present modeling framework incorporates the exchange and magnetostatic energies, and aspects of the magnetoelastic energy have been employed in [36] to develop magnetoelastic constitutive relations

$$
\begin{aligned}
& \sigma=Y^{M_{\varepsilon}} \varepsilon-Y^{M} \gamma M^{2} \\
& M(H)=\int_{0}^{\infty} \int_{-\infty}^{\infty} \nu\left(H_{c}, H_{I}\right) \bar{M}\left(H+H_{I} ; H_{c}, \xi\right) d H_{I} d H_{c}
\end{aligned}
$$

where $\varepsilon, \sigma$ and $\gamma$ respectively denote a uniaxial strain, stress and coupling coefficient. These relations form the basis for constructing PDE models for magnetostrictive transducers, and the performance of the framework for characterizing the magnetization and strains generated by a Terfenol-D transducer analogous to that depicted in Figure 2 is demonstrated in [36].

Attributes and open research questions pertaining to the framework can be summarized as follows. (i) Minor Loop Closure and After-Effects: For operating regimes in which thermal relaxation processes are negligible, use of the kernel (32) in the macroscopic model (69) guarantees the closure of biased minor loops. Alternatively, use of the kernel (49), which incorporates thermal relaxation, yields nonclosure of biased minor loops in certain regimes as well as after-effects of the type shown in Figure 12. (ii) Noncongruency: As noted in Remark 2 and depicted in Figure 5(a), the model employing the kernel (29) incorporates the noncongruency exhibited by certain materials. (iii) Reversibility: The model employing the kernels (32) or (49) provides reversible behavior after switching due to the nonzero slope of the upper and lower hysteron branches (e.g., see Figure 11). (iv) Accommodation: 
Whereas the framework phenomenologically quantifies certain accommodation processes, the extension of the theory to incorporate energy mechanisms associated with magnetic accommodation has not been completed and is under present investigation. (v) Temperature and Stress-Dependencies: The kernel (29) incorporates certain temperature-dependencies as well as the transition between ferromagnetic and paramagnetic phases; however, its accuracy should be considered limited when quantifying changes over a broad temperature range. Certain extensions to the framework to provide more comprehensive characterization of temperature effects has been developed in [35] in the context of relaxor ferroelectric materials. The framework presented here focuses on constant stress conditions. Initial extensions of the theory to incorporate stress-dependencies in $M$ due to magnetoelastic coupling are provide in [73]. (vi) Eddy Currents: The present framework does not incorporate eddy currents and hence should be employed in low frequency regimes or transducers constructed for minimal eddy losses (e.g., laminates). (vii) Anisotropy: The model is presently formulated for isotropic or weakly anisotropic materials and the extension of the framework to incorporate the energy associated with hexagonal and cubic crystalline anistropies is under investigation.

One of the motivating criteria when constructing the characterization framework was to provide macroscopic models with sufficient efficiency to permit model-based control design. It is illustrated in [71] that the monotonicity inherent to the $H$ - $M$ relation can be exploited to construct highly efficient approximate inverse representations $M^{-1}(H)$ using the discretized model (76). These approximate inverses are then employed as filters to the linear and hysteretic transducers so that prescribed and actual control inputs to the plant approximately coincide. It is illustrated in $[71,72]$ that by linearizing a Terfenol-D transducer in this manner, highly accurate tracking tolerances can be maintained through linear robust control designs while operating in highly nonlinear and hysteretic drive regimes.

\section{Appendix A}

We summarize here the attributes of a Dirac sequence and provide a theorem which establishes that the convolution of Dirac sequences with suitably smooth functions $f$ will limit to a point evaluation of $f$. This theorem is employed in the model development to illustrate the convergence of Gaussian moment distributions to a Dirac distribution in the limit of small relative thermal energies $k T / V$ or increasing control volumes $V$. It also provides a framework employed to demonstrate that models which include thermal after-effects converge to models based on the assumption of negligible thermal energy as control volumes are taken to be arbitrarily large.

Theorem 1. Let $\left\{\phi_{j}\right\}$ be a sequence satisfying the following properties:

(i) $\phi_{j}>0$ for all $j$

(ii) $\int \phi_{j}(y) d y=1$ for all $j$

(iii) Given $\varepsilon, \delta>0$, there exists $j_{0}$ such that

$$
\int_{|y| \geq \delta} \phi_{j}(y) d y<\varepsilon
$$

for all $j \geq j_{0}$.

Let $f$ be piecewise continuous on $\mathbb{R}$, continuous on the interval $[a, b]$, and satisfy the decay property: 
(iv) Given $\varepsilon, \delta>0$, there exists $j_{0}$ such that

$$
\int_{|y| \geq \delta} \phi_{j}(y) f(x-y) d y<\varepsilon
$$

for all $j \geq j_{0}$ and $x \in[a, b]$.

Then for $x \in[a, b], \phi_{k} * f$ converges to $f$; that is

$$
\int \phi_{j}(y) f(x-y) d y \rightarrow f(x)
$$

or

$$
\int \phi_{j}(x-y) f(y) d y \rightarrow f(x) .
$$

Proof. From (ii) it follows that

$$
f(x)=f(x) \int \phi_{j}(y) d y=\int f(x) \phi_{j}(y) d y
$$

so that for $x \in[a, b]$,

$$
\begin{aligned}
\phi_{j} * f(x)-f(x) & =\int \phi_{j}(y) f(x-y) d y-\int \phi_{j}(y) f(x) d y \\
& =\int \phi_{j}(y)[f(x-y)-f(x)] d y .
\end{aligned}
$$

From the continuity of $f$, it follows that for fixed $\varepsilon$, there exists $\delta$ such that

$$
|f(x-y)-f(x)|<\varepsilon
$$

for $|y|<\delta$. For this $\delta$, we write

$$
\begin{aligned}
\left|\phi_{j} * f(x)-f(x)\right| \leq & \int_{|y|<\delta} \phi_{j}(y)|f(x-y)-f(x)| d y+\left|\int_{|y| \geq \delta} \phi_{j}(y) f(x-y) d y\right| \\
& +\int_{|y| \geq \delta} \phi_{j}(y)|f(x)| d y .
\end{aligned}
$$

For sufficiently large $j$, the integral over the region $|y|<\delta$ is bounded by $\epsilon$ due to the continuity of $f$ on $[a, b]$ whereas the second integral is bounded by $\varepsilon$ due to (iv). Finally the third integral is bounded by $\|f\|_{\infty} \varepsilon$, where $\|f\|_{\infty} \equiv \max _{x \in[a, b]}|f(x)|$, thus yielding the desired convergence. The convolution expression follows from a direct change of variables.

We note that a sequence of functions $\left\{\phi_{j}\right\}$ satisfying the properties (i)-(iii) is termed a Dirac sequence on $\mathbb{R}^{1}$. Additionally, if we replace the assumption (iv) by the condition that $f$ is bounded and measurable on $\mathbb{R}$, then Theorem 1 is a 1 -D version of Theorem 3.1 from page 228 of [74].

\section{Acknowledgements}

The research of R.C.S. was supported in part through the NSF grants CMS-0099764, CMS0201560 and in part by the Air Force Office of Scientific Research through the grants AFOSR-F4962001-1-0107 and AFOSR-FA9550-04-1-0203. The work of T.R.B. was supported by the United States Department of Education through a GAANN fellowship and the research of M.J.D. was supported in part by Ohio State University. 


\section{References}

[1] J.C. Maxwell, Electricity and Magnetism, Oxford University Press, Oxford, 1873.

[2] S. Chikazumi, Physics of Ferromagnetism, 2nd Ed., Carendon Press, Oxford, 1997.

[3] B.D. Cullity, Introduction to Magnetic Materials, Addison-Wesley, Reading, MA, 1972.

[4] D.C. Jiles, Introduction to Magnetism and Magnetic Materials, Chapman and Hall, New York, 1991.

[5] S.V. Vonsovskii, Magnetism, Volumes I and II, Translated from Russian by Ron Hardin, John Wiley and Sons, New York, 1974.

[6] I.D. Mayergoyz, Mathematical Models of Hysteresis, Springer-Verlag, New York, 1991.

[7] F. Liorzou, B. Phelps and D.L. Atherton, "Macroscopic models of magnetization," IEEE Transactions on Magnetics, 36(2), pp. 418-428, 2000.

[8] A. Aharoni, Introduction to the Theory of Ferromagnetism, 2nd Ed., Oxford University Press, Oxford, 2000.

[9] E. Della Torre, Magnetic Hysteresis, IEEE Press, New York, 1999.

[10] D.C. Jiles and D.L. Atherton, "Theory of the magnetisation process in ferromagnetics and its application to the magnetomechanical effect," Journal of Physics D: Applied Physics, 17, pp. 1265-1281, 1984.

[11] D.C. Jiles and D.L. Atherton, "Theory of ferromagnetic hysteresis," Journal of Magnetism and Magnetic Materials, 61, pp. 48-60, 1986.

[12] D.C. Jiles, J.B. Thoelke and M.K. Devine, "Numerical determination of hysteresis parameters for the modeling of magnetic properties using the theory of ferromagnetic hysteresis," IEEE Transactions on Magnetics, 28(1), pp. 27-35, 1992.

[13] D.C. Jiles, "Modelling the effects of eddy current losses on frequency dependent hysteresis in electrically conducting media," IEEE Transactions on Magnetics, 30(6), pp. 4326-4328, 1994.

[14] D.C. Jiles, A. Ramesh, Y. Shi and X. Fang, "Application of the anisotropic extension of the theory of hysteresis to the magnetization curves of crystalline and textured magnetic materials," IEEE Transactions on Magnetics, 33(5), pp. 3961-3963, 1997.

[15] K.H. Carpenter, "A differential equation approach to minor loops in the Jiles-Atherton hysteresis model," IEEE Transactions on Magnetics, 27(6), pp. 4404-4406, 1991.

[16] D.C. Jiles, "A self-consistent generalized model for the calculation of minor loop excursions in the theory of hysteresis." IEEE Transactions on Magnetics, 28(5), pp. 2602-2604, 1992.

[17] E.C. Stoner and E.P. Wohlfarth, "A mechanism of magnetic hysteresis in heterogeneous alloys," Philosophical Transactions of the Royal Society of London, 240A, pp. 599-642, 1948.

[18] E.W. Lee and J.E.L. Bishop, "Magnetic behavior of single-domain particles," Proceedings of the Physical Society, 89, pp. 661-675, 1966. 
[19] W.D. Armstrong, "Magnetization and magnetostriction processes in $\mathrm{Tb}_{0.27-0.30} \mathrm{Dy}_{0.73-0.70}$ Fe1.9-2.0," Journal of Applied Physics, 81(5), pp. 2321-2326, 1997.

[20] A.E. Clark, H.T. Savage and M.L. Spano, "Effect of stress on magnetostriction and magnetization of single crystal $\mathrm{Tb}_{.27} \mathrm{Dy}_{.73} \mathrm{Fe}_{2}$," IEEE Transactions on Magnetics, 20, pp. 1443-1445, 1984.

[21] D.C. Jiles and J.B. Thoelke, "Theoretical modelling of the effects of anisotropy and stress on the magnetization and magnetostriction of $\mathrm{Tb}_{0.3} \mathrm{Dy}_{0.7} \mathrm{Fe}_{2}$," Journal of Magnetism and Magnetic Materials, 134, pp. 143-160, 1994.

[22] D.L. Atherton and J.R. Beattie, "A mean field Stoner-Wohlfarth hysteresis model," IEEE Transactions on Magnetics, 26(6), pp. 3059-3063, 1990.

[23] B.F. Phelps, F. Liorzou and D.L. Atherton, "Inclusive model of ferromagnetic hysteresis," IEEE Transactions on Magnetics, 38(2), pp. 1326-1332, 2002.

[24] A. Globus and P. Duplex, "Separation of susceptibility mechanisms for ferrites of low anisotropy," IEEE Transactions on Magnetics, 2, pp. 441-445, 1966.

[25] F. Preisach, "Uber die magnetische nachwirkung," Zeitschrift fur Physik, 94, pp. 277-302, 1935.

[26] M. Brokate and J. Sprekels, Hysteresis and Phase Transitions, Springer-Verlag, New York, 1996.

[27] M.E. Shirley and R. Venkataraman, "On the identification of Preisach measures," Proceedings of the SPIE, Smart Structures and Materials 2003, Modeling, Signal Processing, and Control, Vol. 5049, pp. 326-336, 2003.

[28] X. Tan, R. Venkataraman and P.S. Krishnaprasad, "Control of hysteresis: Theory and experimental results," Smart Structures and Materials 2001, Modeling, Signal Processing and Control in Smart Structures, SPIE Vol. 4326, pp. 101-112, 2001.

[29] M.B. Ozer and T.J. Royston, "Modeling the effect of piezoceramic hysteresis in structural vibration control," Proceedings of SPIE, Smart Structures and Materials, Volume 4326, pp. 89-100, 2001.

[30] E. Della Torre and F. Vajda, "Parameter identification of the complete moving hysteresis model using major loop data," IEEE Transactions on Magnetics, 30(6), pp. 4987-5000, 1994.

[31] P. Krejčí and J. Sprekels, "On a system of nonlinear PDEs with temperature-dependent hysteresis in one-dimensional thermoplasticity," Journal of Mathematical Analysis and Applications, 209, pp. 25-46, 1997.

[32] R.C. Smith, Smart Material Systems: Model Development, SIAM, Philadelphia, PA, 2005.

[33] R.C. Smith and S. Seelecke, "An energy formulation for Preisach models," Proceedings of the SPIE, Smart Structures and Materials 2002, Volume 4693, pp. 173-182, 2002.

[34] B.L. Ball, R.C. Smith, S-J. Kim and S. Seelecke, "A stress-dependent hysteresis model for ferroelectric materials," CRSC Technical Report CRSC-TR05-17; Journal of Intelligent Material Systems and Structures, submitted. 
[35] J.K. Raye and R.C. Smith, "A temperature-dependent hysteresis model for relaxor ferroelectric compounds," Proceedings of the SPIE, Smart Structures and Materials 2004, Volume 5383, pp. 1-10, 2004.

[36] R.C. Smith, M.J. Dapino and S. Seelecke, "A free energy model for hysteresis in magnetostrictive transducers," Journal of Applied Physics, 93(1), pp. 458-466, 2003.

[37] E. Ising, "Beitrag zur theorie des ferromagnetismus," Zeitschrift für Physik, 31, pp. 253-258, 1925.

[38] W. Heisenberg, "Zur theorie des ferromagnetismus," Zeitschrift für Physik, 49, pp. 619-636, 1928.

[39] H.E. Stanley, Introduction to Phase Transitions and Critical Phenomena, Oxford University Press, Oxford, 1971.

[40] L.D. Landau and E.M Lifshitz, "On the theory of the dispersion of magnetic permeability in ferromagnetic bodies," Physikalische Zeitschrift der Sowjetunion, 8, pp. 153-169, 1935.

[41] W.F. Brown, Jr., Magnetostatic Principles in Ferromagnetism, North-Holland Publishing Company, Amsterdam, 1962.

[42] W.F. Brown, Jr., Magnetoelastic Interactions, Springer-Verlag, Berlin, 1966.

[43] W.F. Brown, Jr., "Domain, micromagnetics, and beyond, reminiscences and assessments, Journal of Applied Physics, 49, pp. 1937-1942, 1978.

[44] W. Williams and D.J. Dunlop, "Three-dimensional micromagnetic modelling of ferromagnetic domain structure," Nature, 337, pp. 634-637, 1989.

[45] F.T. Calkins, R.C. Smith and A.B. Flatau, "An energy-based hysteresis model for magnetostrictive transducers," IEEE Transactions on Magnetics, 36(2), pp. 429-439, 2000.

[46] M.J. Dapino, R.C. Smith, L.E. Faidley and A.B. Flatau, "A coupled structural-magnetic strain and stress model for magnetostrictive transducers," Journal of Intelligent Material Systems and Structures, 11(2), pp. 134-152, 2000.

[47] M.J. Dapino, R.C. Smith and A.B. Flatau, "A structural-magnetic strain model for magnetostrictive transducers," IEEE Transactions on Magnetics, 36(3), pp. 545-556, 2000.

[48] W. Gorsky, "Röntgenugraphische untersuchung von umwandlungen in der legierung CuAu," Zeitschrift für Physik, 50, pp. 64-81, 1928.

[49] W.L. Bragg and E.J. Williams, "The effect of thermal agitation on atomic arrangement in alloys," Proceedings of the Royal Society of London, A145, pp. 699-730, 1934.

[50] W.L. Bragg and E.J. Williams, "The effect of thermal agitation on atomic arrangement in alloys II," Proceedings of the Royal Society of London, A151, pp. 540-566, 1935.

[51] E.J. Williams, "The effect of thermal agitation on atomic arrangement in alloys III," Proceedings of the Royal Society of London, A152, pp. 231-252, 1935.

[52] D. ter Haar, Elements of Statistical Mechanics, 3rd Ed., Butterworth-Heinemann, Oxford, 1995. 
[53] R.K. Pathria, Statistical Mechanics, Pergamon Press, Oxford, 1972.

[54] R.C. Smith and J.E. Massad, "A unified methodology for modeling hysteresis in ferroic materials," Proceedings of the 2001 ASME Design Engineering Technical Conferences and Computers and Information in Engineering Conference, Vol 6, Pt B, pp. 1389-1398, 2001.

[55] R.C. Smith and C.L. Hom, "Domain wall theory for ferroelectric hysteresis," Journal of Intelligent Material Systems and Structures, 10(3), pp. 195-213, 1999.

[56] H.T. Banks, A.J. Kurdila and G. Webb, "Identification of hysteretic control influence operators representing smart actuators Part I: Formulation," Mathematical Problems in Engineering, 3, pp. 287-328, 1997.

[57] H.T. Banks, A.J. Kurdila and G. Webb, "Identification of hysteretic control influence operators representing smart actuators Part II: Convergent approximations," Journal of Intelligent Material Systems and Structures, 8(6), pp. 536-550, 1997.

[58] M.A. Krasnosel'skiř and A.V. Pokrovskiǐ, Systems with Hysteresis, Nauka, Moscow, 1983; Translated by M. Niezgódka, Springer-Verlag, Berlin, 1989.

[59] D.J. Craik and R.S. Tebble, Ferromagnetism and Ferromagnetic Domains, North-Holland Publishing Company, Amsterdam, 1965.

[60] R.C. Smith and A. Hatch, "Parameter estimation techniques for nonlinear hysteresis models," Smart Structures and Materials 2004, Proceedings of the SPIE, Volume 5383, pp. 155-163, 2004.

[61] R.C. Smith, A. Hatch, B. Mukherjee and S. Liu, "A homogenized energy model for hysteresis in ferroelectric materials: General density formulation," CRSC Technical Report CRSC-TR04-23; Journal of Intelligent Material Systems and Structures, to appear.

[62] D. Zwillinger, Editor-in-Chief, CRC Standard Mathematical Tables and Formulae, 30th Edition, CRC Press, Boca Raton, 1996.

[63] R.C. Smith, S. Seelecke, M.J. Dapino and Z. Ounaies, "A unified model for hysteresis in ferroic materials," Proceedings of the SPIE, Smart Structures and Materials 2003, Volume 5049, pp. 8899, 2003.

[64] R.C. Smith, S. Seelecke, M.J. Dapino and Z. Ounaies, "A unified framework for modeling hysteresis in ferroic materials," CRSC Technical Report CRSC-TR04-35; Journal of the Mechanics and Physics of Solids, submitted.

[65] A.P. Mortensen, "Characterization, modeling and dynamic implementation of Terfenol-D particulate composites," M.S. Thesis, The Ohio State University, Columbus, OH, 2005.

[66] J.E. Massad, R.C. Smith and G.P. Carman, "A free energy model for thin-film shape memory alloys," Proceedings of the SPIE, Smart Structures and Materials 2003, Volume 5049, pp. 13-23, 2003.

[67] S. Seelecke, "Modeling the dynamic behavior of shape memory alloys," International Journal of Non-Linear Mechanics, 37, pp. 1363-1374, 2002.

[68] S. Seelecke and C. Büskens, "Optimal control of beam structures by shape memory wires," in OPTI 97, Computer Aided Optimum Design of Structures, Rome, Italy, Sept. 8-10, 1997, (eds., S. Hernandez and C.A. Brebbia), Computational Mechanics Press, Rome, Italy, 1997. 
[69] S. Seelecke and I. Müller, "Shape memory alloy actuators in smart structures - Modeling and simulation," ASME Applied Mechanics Reviews, 57(1), pp. 23-46, 2004.

[70] R.C. Smith, S. Seelecke, Z. Ounaies and J. Smith, "A free energy model for hysteresis in ferroelectric materials," Journal of Intelligent Material Systems and Structures, 14(11), pp. 719-739, 2003.

[71] J.M. Nealis and R.C. Smith, "H $\mathcal{H}_{\infty}$ control design for a magnetostrictive transducer," Proceedings of the 42nd IEEE Conference on Decision and Control, Maui, HA, pp. 1801-1806, 2003.

[72] J.M. Nealis and R.C. Smith, "Model-based robust control design for magnetostrictive transducers operating in hysteretic and nonlinear regimes," CRSC Technical Report CRSC-TR03-25; IEEE Transactions on Control System Technology, submitted.

[73] R.C. Smith and M.J. Dapino, "A stress-dependent model for ferromagnetic compounds," 2005 ASME International Mechanical Engineering Congress and Exposition, Orlando, FL, submitted.

[74] S. Lang, Real and Functional Analysis, Springer-Verlag, New York, 1993. 